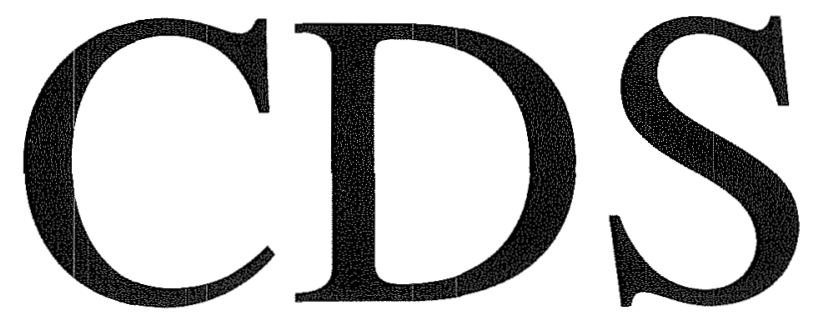

Technical Memorandum No. CIT-CDS 93-001

January 28, 1993

"Multiple Steady States in Homogeneous
Azeotropic Distillation"
Nikolaos Bekiaris, George A. Meski, Christian M. Radu, and Manfred Morari

Control and Dynamical Systems California Institute of Technology

Pasadena, CA 91125 


\title{
Multiple Steady States in Homogeneous Azeotropic Distillation
}

\author{
Nikolaos Bekiaris \\ George A. Meski \\ Cristian M. Radu \\ Manfred Morari* \\ Chemical Engineering 210-41 \\ California Institute of Technology \\ Pasadena, CA 91125
}

CIT/CDS Technical Report No. 93-001

January 28, 1993

\begin{abstract}
In this article we study multiple steady states in ternary homogeneous azeotropic distillation. We show that in the case of infinite reflux and an infinite number of trays one can construct bifurcation diagrams on physical grounds with the distillate flow as the bifurcation parameter. Multiple steady states exist when the distillate flow varies non-monotonically along the continuation path of the bifurcation diagram. We derive a necessary and sufficient condition for the existence of these multiple steady states based on the geometry of the distillation region boundaries. We also locate in the composition triangle the feed compositions that lead to these multiple steady states. We further note that most of these results are independent of the thermodynamic model used. We show that the prediction of the existence of multiple steady states in the case of infinite reflux and an infinite number of trays has relevant implications for columns operating at finite reflux and with a finite number of trays. Using numerically constructed bifurcation diagrams for specific examples, we show that these multiplicities tend to vanish for small columns and/or for low reflux flows. Finally, we comment on the effect of multiplicities on column design and operation for some specific examples.

KEYWORDS: distillation, azeotropic distillation, multiplicity, distillation design, extractive distillation.
\end{abstract}

*Author to whom correspondence should be addressed. Phone: (818) 356-4186. Fax: (818) 568-8743. E-mail: MM@IMC.CALTECH.EDU. 


\section{Introduction}

Azeotropic distillation is one of the most widely used and most important separation operations in the chemical and the specialty chemical industry. Despite that, the design and operation of azeotropic distillation columns are relatively poorly understood and little studied. Among their surprising features, it has been discovered that such columns can exhibit multiple steady states i.e. two or more steady states with different composition and temperature profiles which correspond to the same set of operating parameters. In this article we are only investigating this type of multiplicities.

The study of multiplicities in distillation has a long history. Rosenbrock (1962) proved that the steady state of distillation columns separating a binary mixture is unique under the assumptions of (1) constant molar flows (i.e. neglecting the energy balances) and (2) that to every value of vapor composition $y$ there corresponds a unique value of liquid composition $x$ in equilibrium with $y$. This assumption does not exclude the cases of nonideal vapor-liquid equilibrium (including the cases where an azeotrope is formed between the two components).

Petlyuk and Avetyan (1971) first conjectured the possibility of multiple steady states in the distillation of ternary homogeneous systems under the assumptions of constant molar flows and nonideal vapor-liquid equilibrium (Wilson equation). They conjectured that multiple steady states exist when a distillation product region is a quadrangle. However, as we will show this condition is neither necessary nor sufficient for the existence of multiple steady states. Moreover, they do not identify any physical mixture that may lead to these multiple steady states.

Magnussen et al. (1979) present simulation results that show the existence of three steady states for the heterogeneous mixture of ethanol - water - benzene. In these calculations the phase splitter is removed; instead, a second feed at the top of the column is considered (this second feed is the same for all three steady states). Moreover, the liquid composition profiles of all three steady states lie entirely in the single liquid phase region. Therefore, although the mixture ethanol - water - benzene can exhibit liquid - liquid phase split, the multiplicities presented in that article cannot be explained by the heterogeneity of the mixture. Hence, the explanation for the existence of the aforementioned multiplicities should be sought in the regime of homogeneous azeotropic distillation. Finally, it should be noted that the multiplicities were observed with the UNIQUAC and NRTL activity coefficient models but a unique steady state was found with the Wilson equation model.

Doherty and Perkins (1982) considered the case of nonideal vapor - liquid equilibrium and constant molar flows. They proved the stability of the unique steady state in binary distillations (uniqueness was already proven by Rosenbrock, 1962). They also prove that a unique steady state exists for single-staged "columns" of any multicomponent mixture. Using the above results, they conclude that the multiplicity reported by Magnussen et al. (1979) is a consequence of multiple components and multiple stages.

The "discoveries" by Magnussen et al. (1979) triggered great interest in multiple steady states in distillation. The belief that heterogeneity is a possible cause for such multiple steady states directed the attention towards heterogeneous azeotropic distillation. Conse-

quently, several articles were published where the results of Magnussen et al. (1979) were studied extensively and where multiplicities for other heterogeneous systems were reported (Prokopakis and Seider, 1983; Kovach and Seider, 1987; Widagdo et al., 1989). 
However, other types of systems have also been investigated. In a simulation study Chavez et al. (1986) and Lin et al. (1987) found multiple steady states in interlinked distillation columns. The multiplicity they report is due to the interlinking and is not found in single columns.

Sridhar and Lucia (1989) considered binary mixtures with nonideal VLE and included energy balances in the model. They showed that any binary homogeneous separation process, in which the temperature and pressure profiles are specified, has a unique steady state.

Jacobsen and Skogestad (1991) present two different types of multiplicities in binary distillation columns with ideal VLE:

- Multiplicity in Input Transformations.

Constant molar flows are assumed. Multiplicities occur when some flows are specified on a mass basis (instead of a molar basis) and are due to the nonlinear mass to molar flow transformation.

- Multiplicity when molar reflux and boilup are used as specifications (LV configuration).

Energy balances are included in the model. This type of multiplicity does not occur for the case of constant molar flows.

Most recently, Kienle et al. (1992) reported multiple steady states for the ternary homogeneous mixture of acetone, chloroform and methanol. The starting point for the study presented here were the multiple steady states reported by Laroche et al. $(1990,1991,1992)$ for a homogeneous ternary mixture (acetone - heptane - benzene) with nonideal VLE and constant molar flows.

\section{Background}

The term "homogeneous azeotropic distillation" covers the general notion of distillation of azeotrope forming mixtures where a single liquid phase exists in the region of interest. Usually, homogeneous azeotropic distillation units perform the separation of a binary azeotrope into two pure components through the addition of an entrainer which alters the relative volatility of the two azeotrope constituents without inducing liquid - liquid phase separation.

Unless stated otherwise, we use the following convention to refer to a given mixture: L (I, $\mathrm{H}$ respectively) corresponds to the component which has the lowest (intermediate, highest resp.) boiling point; we also denote the entrainer by $\mathrm{E}$. We use the same notation in italics $(L, I, H, E)$ to denote the corresponding flow rates of the components in the feed. The locations of the feed, distillate and bottoms in the composition triangle are denoted by $F, D$ and $B$ respectively. Again, the corresponding flowrates are denoted by the same letters in italics ( $F, D, B$ and $R$ for the reflux flow).

In all simulations presented in this paper, the column operates under atmospheric pressure, there is no pressure drop in the column and the condenser is total. Moreover, constant molar overflow and a tray efficiency of 1 are assumed. Vapor pressures are calculated using the Antoine equation and liquid activity coefficients are calculated using the Van Laar equation. The appendix contains more information on the thermodynamic model as well as the 
Antoine and Van Laar coefficients used in the examples. The tray counting starts from the reboiler (number 0 ) and ends at the top. Finally, in all composition profile figures the liquid mole fractions are depicted.

A widely used concept for the description of azeotropic distillation is that of the simple distillation residue curve (hereafter called residue curve). The simple distillation process involves charging a still with a liquid of composition $\underline{x}$ and gradual heating. The vapor formed is in equilibrium with the liquid left in the still; the vapor is continuously removed from the still.

A residue curve is defined as the locus of the composition of the liquid remaining at any given time in the still of a simple distillation process. Residue curves are governed by the set of differential equations (Doherty and Perkins, 1978):

$$
\frac{d x_{i}}{d \xi}=x_{i}-y_{i}, \quad i=1, \ldots, C-1
$$

where $i$ is the component index, $C$ is the number of pure components in the mixture, $y_{i}\left(x_{i}\right)$ is the mole fraction of component $i$ in the vapor (liquid) phase, and $\xi$ is the dimensionless warped time.

At infinite reflux, the differential equations which describe packed columns become identical to the residue curve equations. Thus residue curves coincide exactly with composition profiles of packed columns operated at total reflux, and they give a very good approximation of composition profiles of tray columns at infinite reflux.

A distillation region is a subset of the composition simplex in which all residue curves originate from a locally lowest-boiling pure component or azeotrope and travel toward a locally highest-boiling one. The curves which separate different distillation regions are called residue curve boundaries. The term distillation region boundary (or just boundary) is used for both residue curve boundaries (interior boundaries) and for the edges of the composition simplex.

\section{Infinite Reflux and Infinite Number of Trays}

In this section we present an extensive analysis of the case where the reflux and the number of trays are infinite (the $\infty / \infty$ case hereafter). The idea for examining this situation came from the multiplicities reported by Laroche et al. (1990, 1991, 1992). The homogeneous mixture under consideration is that of acetone $(\mathrm{L})$, heptane $(\mathrm{H})$ and benzene $(\mathrm{I})$. In this case there is only one binary azeotrope formed between acetone and heptane (93\% acetone, $7 \%$ heptane). Benzene, the intermediate boiler, is used as entrainer for the separation of the acetone - heptane azeotrope. Figure 1 shows the residue curve map of this ternary mixture (001 class according to the classification by Matsuyama and Nishimura, 1977).

Figure 2 depicts the separation sequence and information about the azeotropic column. The feed composition and flows, the number of trays and the distillate, bottom, reflux and reboil flow rates are identical for both steady states. Figure 3 shows the two different stable steady state profiles reported by Laroche et al. $(1990,1991,1992)$. In the first case (Figure $3 \mathrm{a}$ ) the column yields $99 \%$ acetone (L) at the top and $95 \%$ heptane $(\mathrm{H})$ at the bottom while in the second case (Figure $3 \mathrm{~b}$ ), the top product is a mixture of $93 \%$ acetone and $7 \%$ heptane (azeotropic mixture). 
In this column, the reflux to feed and the reflux to distillate flow ratios are very high - in the order of 100. Table 1 summarizes simulation results with different reflux and associated reboil flows. All other column parameters are kept constant at their values shown in Figure 2. Table 1 shows that only one stable steady state exists for reflux flows less than 6600 while two stable steady states are observed for any higher reflux. Actually, no matter how large a reflux flow was used, two stable steady states were always found. This result suggests that this type of multiplicity may also occur at infinite reflux. This observation simplifies the study of multiplicities significantly since at infinite reflux column profiles coincide with residue curves.

Moreover, the column shown in Figure 2 has 64 theoretical trays which is quite a large number. This suggests that this multiplicity may occur in columns with a large number of trays (infinite number of trays in the limit). Columns at infinite reflux and with an infinite number of trays are obviously a special case of infinite-reflux columns which simplifies our analysis even further.

\subsection{Existence of Multiple Steady States}

In this section we study in detail the $\infty / \infty$ case. We use a 001 class ternary mixture to illustrate the analysis of this situation. Figure 4 shows the residue curve map of this type of ternary mixture. In this diagram, there is only one minimum boiling binary azeotrope between the light $(\mathrm{L})$ and the heavy $(\mathrm{H})$ component. The azeotrope is an unstable node, the light and the intermediate pure component corners are saddles and the heavy-component corner is a stable node. All residue curves start from the azeotrope and end at the heavy component corner; there are no interior distillation boundaries in this diagram and hence the whole triangle forms a single distillation region.

At infinite reflux, column profiles coincide with residue curves. In the special case of columns with an infinite number of trays there is one additional requirement: The column profile should include a pinch point. There are four candidate pinch points in the residue curve map shown in Figure 4, namely the three pure component corners and the azeotrope. Therefore, in the $\infty / \infty$ case, the only acceptable columns belong to one of the following types:

I. Columns whose distillate composition is that of the azeotrope (unstable node). In this case, the column profile starts from the azeotrope (top of the column), follows a residue curve and ends at an arbitrary point on the same residue curve (bottom product).

II. Columns whose bottom product composition is pure heavy component (stable node). In this case, the column profile starts from an arbitrary point in the composition triangle, follows the residue curve that passes through this starting point and ends at the heavy component corner (bottom product).

III. Columns whose composition profiles run along the edges of the triangle and contain at least one of the saddle corners (light and intermediate component corners). In this case, the top and bottom products lie on the edges of the triangle.

In the $\infty / \infty$ case, given a feed composition and a feed flowrate $F$, the only unspecified parameter is the distillate flow rate $D$ (the bottom flow rate is $B=F-D$ from the overall 
material balance). In order to find whether multiple steady states can occur (i.e. whether different column profiles correspond to the same value of $D$ ) we find all possible composition profiles by tracking the distillate and bottoms in the composition triangle, starting from the column profile with $D=0$ and ending with the column profile with $D=F$. That is, we perform a bifurcation study (continuation of solutions) using the distillate flow as the bifurcation parameter. This task can be achieved because in the $\infty / \infty$ case a continuation of solutions can be carried out based on physical arguments only. The light component mole fraction in the distillate $x_{D L}$ is recorded along this "continuation path." The analysis that will follow can be applied to any feed composition but just for simplicity, we assume a feed that lies on the line connecting the azeotrope and the corner I. Therefore, $F=L+I+H$ and $L /(L+H)$ equals the azeotropic composition of the light component.

If $D=0$ then $B=F=L+I+H$ and therefore the composition of the bottom product coincides with that of the feed $F$. Hence the bottom product composition is an interior point of the composition triangle (i.e. it does not lie on an edge). The only acceptable column profile (as defined above) that ends (bottom product) at an interior point of the triangle is the one that starts (top of the column) from the azeotrope and follows the residue curve that the bottoms composition lies on. This is a type I column profile. Figure 5a shows the column profile for $D=0$. Therefore, in this case, $x_{D L}=L /(L+H)$, the azeotropic composition.

Using this as a starting profile, we will find all possible type I column profiles for the given feed. Since, for this type of profile, the top of the column coincides with the azeotrope, the material balance line is a segment of the line connecting the azeotrope, the feed and the intermediate component corner (for this particular choice of feed composition). Therefore the bottom composition (B) can be any point on the line segment between the feed $F$ and the intermediate component corner (I). Figure 5b illustrates a type I column profile with the aforementioned characteristics. As B moves along the FI line segment from $F$ to $I$ the line BF continuously lengthens. Therefore, according to the lever material balance rule, the bottoms flow decreases monotonically from the initial $F$ to $I$ while the distillate flow will increase monotonically from initially 0 to $L+H$. The composition of $\mathrm{L}$ in the distillate $\left(x_{D L}\right)$ for all type I column profiles is kept constant and equal to the azeotropic composition $(L /(L+H))$. Therefore, a column profile of type I (similar to that of Figure $5 \mathrm{~b}$ ) exists for $0<D<H+L$.

Figure 5c shows the profile with the bottoms composition B located at the intermediate component corner (I) and the distillate composition located at the azeotrope. In this case, $D=H+L$ and $B=I$. Both $\mathrm{B}$ and $\mathrm{D}$ lie on an edge of the composition triangle and therefore in this case the column profile belongs to type III. Using this as a starting profile, we will find all possible type III column profiles for the given feed.

In this type of profiles, both $\mathrm{D}$ and $\mathrm{B}$ must lie on the edges of the triangle. There exist two alternative routes: $B$ should move along either the IL edge or the IH edge. In the first case, the material balance implies that $D$ has to move on the line segment between the azeotrope and the heavy component corner $(\mathrm{H})$. This is not allowable though because there is no residue curve connecting $\mathrm{D}$ and $\mathrm{B}$. In the second case, $\mathrm{D}$ has to move along the line segment between the azeotrope and the light component corner while B lies on the IH edge. In this case, there exists a residue curve connecting $B$ and $D$.

Figure 5d illustrates such a column profile. Since D lies on the LH edge the composition of the intermediate component $I$ in the distillate is zero and therefore the whole amount 
of $\mathrm{I}$ fed into the column is recovered in the bottom product. Because $\mathrm{B}$ lies on the $\mathrm{IH}$ edge, there exists some amount of heavy component in the bottom product while the whole amount of $\mathrm{L}$ fed is recovered entirely in the distillate. Therefore $B>I$ and consequently $D<H+L$. As $\mathrm{D}$ moves along the $\mathrm{LH}$ side from the azeotrope to the light component corner, the amount of the heavy component in the distillate decreases and consequently the distillate flow decreases monotonically from $L+H$ to $L$ (when $\mathrm{D}$ is located at the light component corner). Therefore, a column profile of type III similar to that shown in Figure 5d exists for $L+H>D>L$. Since all the light component fed is recovered in the distillate, $x_{D L}=L / D$. Therefore along this part of the continuation path, the light component concentration in the distillate increases monotonically from $L /(L+H)$ to 1 .

Figure 5e shows the profile with the distillate composition $D$ located at the light component corner (L). In this case $D=L$ and $B=I+H$. As B moves further along the IH side towards the $\mathrm{H}$ corner, $\mathrm{D}$ moves along the LI edge towards the corner I. Figure $5 \mathrm{f}$ illustrates such a type III column profile. In this case, D contains no heavy component, some amount of the intermediate component and all the light component fed. Consequently, B contains no light component, some amount of the intermediate component and all the heavy component fed into the column. As $\mathrm{B}$ moves along the $\mathrm{IH}$ edge towards the heavy component corner the bottom product flow decreases monotonically from the initial $I+H$ to $H$ (when $\mathrm{B}$ is located at the $\mathrm{H}$ corner). Consequently along this part of the continuation path the distillate flow increases monotonically from $L$ to $L+I$. Therefore a column profile of type III similar to that shown in Figure $5 f$ exists for $H<D<I+H$. Along this part of the continuation path, $x_{D L}=L / D$ and hence $x_{D L}$ decreases monotonically from 1 to $L /(L+I)$.

Figure 5g shows the column profile with the bottoms composition $B$ located at the heavy component corner $(\mathrm{H})$. In this case $B=H, D=L+I$ and $x_{D L}=L /(L+I)$. B is not allowed to move along the HL edge because a residue curve connecting $B$ and $D$ does not exist. Therefore all type III profiles have been found. The last case to be examined is the type II profiles. In this case the bottoms product composition is $100 \%$ heavy component ( $\mathrm{H}$ corner). Therefore, the material balance line lies on the line connecting the feed $\mathrm{F}$ and the heavy component corner $\mathrm{H}$. Hence, the distillate composition $\mathrm{D}$ can be any point on this line between the feed $\mathrm{F}$ and the LI edge. Figure 5h shows a type II column profile with the aforementioned characteristics. As D moves towards F, the length of DF decreases. Therefore, according to the lever material balance rule, the distillate flow increases monotonically from the initial $I+L$ to $I+L+H(=F)$ while the bottoms flow decreases from $H$ to zero. Therefore a column profile of type II similar to that shown in Figure $5 \mathrm{~h}$ exists for $I+L<D<I+L+H=F$. Along this part of the continuation path, the composition of $\mathrm{L}$ in the distillate decreases from $L /(L+I)$ to $L /(L+I+H)$ according to the rule $x_{D L}=L / D$. Finally, the endpoint of this exhaustive search for all possible column profiles is the column profile with $D=F, B=0$ and $x_{D L}=x_{F L}$.

Now, we put all these pieces together by recording $x_{D L}$ vs. $D$ in a diagram (Figure 6). In the begining as $D$ increases from zero to $L+H, x_{D L}$ remains constant at $L /(L+H)$ (the azeotropic composition). Then $D$ decreases from $L+H$ to $L$ while $x_{D L}=L / D$ and therefore increases from $L /(L+H)$ to 1 . Then $D$ increases again from $L$ to $L+I$ and finally to $F$ while $x_{D L}=L / D$ and hence decreases from 1 to $L /(L+I)$ and finally to $L / F$ (the feed composition). For illustrative purposes only, in Figure 6 we draw two separate curves, ce and eh, although they are actually coinciding $\left(x_{D L}=L / D\right)$. Points $\mathrm{a}-\mathrm{h}$ in Figure 
6 correspond to the column profiles shown in Figures 5a - 5h. Figure 6 shows that for $D$ between $L$ and $L+H$ there exist three steady states (points $1-3$ ):

- Point 1 always corresponds to a column profile of type I like the one depicted in Figure $5 \mathrm{~b}$.

- Point 2 always corresponds to a type III column profile where the distillate composition lies on the line segment between the azeotrope and L (similar to Figure 5d).

- For point 3 there are two cases:

- If $I<H$ and $D>L+I$ then point 3 corresponds to a type II column profile (similar to that of Figure $5 \mathrm{~h}$ ).

- In the case that $I>H$ (Figure 6) as well as in the case that $I<H$ but $D<L+I$, point 3 corresponds to a type III column profile where the distillate composition lies on the LI edge (similar to that in Figure 5f).

In our analysis, a special choice of feed composition has been used. It is very simple to apply the same procedure to any feed composition and prove that for any feed composition inside the composition triangle three steady states exist. Therefore, for this class of residue curve diagrams, namely the 001 class, three steady states exist for any feed composition. Moreover, in this case the existence of multiplicities is independent of the thermodynamic model used to describe the vapor - liquid equilibrium.

Given any ternary mixture, its residue curve diagram and a feed composition, it is very simple to conclude whether multiple steady states can occur in the $\infty / \infty$ case by applying the procedure described above. Next, we examine the key issues that lead to the existence of these multiple steady states.

\subsection{Analysis}

In the previous section, we tracked a "path" generating all possible column profiles starting from the column profile with $D=0$ (type I) and ending at the column profile with $D=F$ (type II). In the beginning $D$ increases, then decreases and then increases again. The key feature that brought about the multiple steady states is that in a segment along this "path"

$D$ decreased. Therefore, in order to find rules for the existence of multiple steady states, we have to first answer why $D$ decreased along the continuation path.

In this section, we assume that distillation boundaries are straight lines (this assumption will be dropped later). Therefore, any distillation region containing $\mathbf{n}(\mathbf{n} \geq 3)$ singular points is an $\mathbf{n}$-polygon. In every distillation region there is one unstable node (the origin of all residue curves in the region), one stable node (the endpoint of all residue curves in the region) and $n-2$ saddles. Finally, we assume that $\mathrm{F}$ is an interior point of a distillation region. It is easy to show that for feeds on a distilation region boundary, $D$ cannot decrease along the continuation path.

Using arguments similar to those in the previous section, it is easy to show that along the continuation path, first we track all possible type I column profiles, then those of type III and last all type II column profiles. Moreover, again using the arguments which were discussed in the previous section, it can be proven that: 
Fact 1 Along the continuation path, $D$ increases monotonically as we track all type I and type II column profiles.

Therefore, a decrease in $D$ can only occur as we track the type III column profiles i.e. columns whose composition profiles run along the edges of the distillation region where $F$ is located and contain at least one of the saddle singular points. In this case, the top and bottom products lie on the edges of the distillation region. Next we will show the following:

Fact 2 Along the continuation path, $D$ increases monotonically for all type III column profiles that contain only one saddle singular point.

Figure 7 shows a column profile (DsB) that contains only one saddle point (s). The lines $\mathrm{ds}$ and $\mathrm{sb}$ are distillation region boundaries. The arrows on ds and $\mathrm{sb}$ show the direction of the residue curves; this direction coincides with the direction of the continuation path. $\mathrm{D}^{\prime} \mathrm{sB}^{\prime}$ is another, "later," column profile along this path. We examine what happens to $D$ as we move from $\mathrm{DsB}$ to $\mathrm{D}^{\prime} \mathrm{sB}^{\prime}$.

Draw the line that is parallel to $\mathrm{BB}^{\prime}$ and passes through $\mathrm{D}$. Name $\mathrm{D}^{\prime \prime}$ the point where this line intersects the $D^{\prime} B^{\prime}$ line. By construction, $F B / D F=F B^{\prime} / D^{\prime \prime} F$. Since $D^{\prime \prime} F>D^{\prime} F$ then $\mathrm{FB} / \mathrm{DF}<\mathrm{FB}^{\prime} / \mathrm{D}^{\prime} \mathrm{F}$. Therefore by the lever material balance rule, we conclude that $D$ increases along the continuation path. This result is independent of the angle dsb, and therefore $D$ increases monotonically for all type III column profiles that contain only one saddle singular point. Q.E.D.

Note that fact 2 is equivalent to the following:

Fact $3 A$ decrease in $D$ can only occur as we track type III column profiles that contain at least two saddles.

Two consequences of fact 3 are:

(1) If multiplicities exist, one of the multiple steady state profiles will contain at least two saddles.

(2) A necessary condition for the existence of this type of multiplicities is that the residue curve diagram contains at least two neighboring saddles.

The situation of at least two neighboring saddles arises in 77 out of the 113 possible residue curve diagrams (as classified by Matsuyama and Nishimura, 1977). Among the residue curve diagrams that do not contain two neighboring saddles are the ideal case $(000$ class) and the case of a heavy entrainer that does not introduce any additional azeotropes (100 class) which are depicted in Figure 8. Note also that no more than three steady states can exist in the case of two neighboring saddles while for certain feed compositions it is possible that more than three steady states exist in the case of more than two neighboring saddles.

However, the condition of at least two neighboring saddles is not sufficient for the existence of multiple steady states. There are two additional requirements. 


\subsubsection{Geometry of the Distillation Boundaries}

The existence of multiplicities depends on the geometry of the distillation boundaries that form the two saddles. - Figures $9 \mathrm{a}$ and $9 \mathrm{~b}$ illustrate two cases of two neighboring saddles. The only difference between the two is the orientation of the ds distillation boundary. In order to check if $D$ increases or decreases along the continuation path, the procedure used for the proof of fact 2 is applied.

In Figure $9 b$, the line from $D$ that is parallel to $B B^{\prime}$ crosses the $D^{\prime} B^{\prime}$ line segment while it does not cross it in Figure 9a. Hence in Figure 9a, $D^{\prime \prime} F>D^{\prime} F$ while $D^{\prime \prime} F<D^{\prime} F$ in Figure $9 b$. As a result $D$ increases in Figure 9a whereas $D$ decreases in Figure $9 \mathrm{~b}$. Therefore multiple steady states exist only for the situation depicted in Figure $9 \mathrm{~b}$. Note that the existence of multiple steady states depends on the relative position of the boundaries ds and s'b while the location of the ss' boundary does not play any role.

In summary, for the existence of multiplicities it is required that (geometrical condition): As we move along the continuation path from $D$ to $D^{\prime}$ and accordingly from $B$ to $B^{\prime}$, the line that passes from $\mathrm{D}$ and is parallel to $B \mathrm{~B}^{\prime}$ crosses the $\mathrm{D}^{\prime} \mathrm{B}^{\prime}$ line segment.

\subsubsection{Appropriate Feed Composition}

Even if a residue curve diagram contains two neighboring saddles with the appropriate geometry (as described above) for the existence of multiplicities, there might be some feed compositions for which multiple steady states do not exist. Figure 10 shows a residue curve diagram that belongs in the 231 class. In this diagram there are two distillation regions. In the lower region there are three saddles (two of them neighboring) while in the upper region there is only one saddle. Therefore if the feed composition lies in the upper region, a unique steady state exists for each value of $D$. However, placing the feed in the lower region is not sufficient for the existence of multiple steady states.

As it can be seen from Figure 10, ab and Ic form the only pair of boundaries that enables the existence of multiple steady states. Hence, the only feed compositions that will exhibit multiple steady states are those that can be separated in a distillate lying on ab and a bottom product lying on Ic for some value of $D$. Therefore, multiple steady states exist for any feed located in the convex hull formed by ab and Ic (shaded region in Figure 10).

In summary, multiple steady states exist only for the feed compositions that lie in the convex hull formed by a pair of distillation region boundaries that satisfy the geometrical condition described above.

\subsubsection{Summary}

In this section we studied the $\infty / \infty$ case for a ternary mixture under the assumption of straight line boundaries. We found a necessary condition for the existence of multiplicities (at least two neighboring saddles). Furthermore, the conditions developed above for the geometry of the boundaries and the appropriate feed compositions constitute a necessary and sufficient condition for the existence of multiple steady states in $\infty / \infty$ case.

Although we assumed that the line connecting two singular points (distillation boundary) is straight, fact 1 is independent of the shape of the boundary. Moreover, the discussion about 
the geometry of the distillation region boundaries and the appropriate feed compositions can be generalized to curved boundaries. This is the topic of the next section.

\subsection{Curved Boundaries}

Distillation region boundaries that do not coincide with the sides of the composition triangle are often curved and in some cases highly curved. The curvature of the boundary may affect the region of feed compositions that lead to multiplicities because the geometry of the boundaries is changed. This is illustrated by Figure 11. This figure is similar to Figure 10 with the difference that the interior boundary ab is curved. However, the location of the azeotropes in the composition triangle is the same in both figures. The following interesting result can be easily shown: if multiple steady states exist under the straight boundaries assumption, then, assuming that the azeotropic compositions do not change, these multiplicities still exist even if the boundaries are curved, although the appropriate feed region is changed.

Point $d$ is the point on the boundary ab where the tangent to the boundary is parallel to Ic. It is apparent that the boundary segment ad and the boundary Ic satisfy the geometry requirement for the existence of multiplicities while bd and Ic do not. Therefore, in this case the appropriate feed location is inside the convex hull formed by ad (not ab) and Ic (shaded region in Figure 11).

In the previous section we concluded that the occurence of two neighboring saddles is a necessary condition for multiplicities when boundaries are straight. This is not true in the general case of curved boundaries, because highly curved boundaries can function as "pseudo-saddles" and therefore can induce multiplicities.

Figure 12 shows a residue curve diagram belonging to the 021 class. In this figure there is a highly curved boundary that separates the composition triangle in two distillation regions. In each region there are two routes which go from the unstable node to the stable node along the region boundaries ( $a$ total of four routes, namely $a \rightarrow L \rightarrow I, a \rightarrow H, a \rightarrow b \rightarrow I, a \rightarrow b \rightarrow H$ ). In the right region there is only one saddle singular point and in the left region there are two saddles but they are not neighboring. Therefore, if the boundary running from $a$ to $b$ were a straight line, there would not exist multiplicities for this mixture. The boundary ab is curved enough so that there exists a point $c$ on it where the tangent to the boundary is parallel to the IH edge.

Now, the geometrical condition can be applied to check for multiplicities. Note that the distillate and bottoms compositions should lie on the same route and therefore we only have to check the geometrical condition along the four routes mentioned above. Also note that the type III column profile with an infinite number of trays should contain a saddle singular point and therefore this constitutes an additional restriction.

The $a \rightarrow b \rightarrow H$ route contains one saddle point (b). The restriction due to the infinite number of trays implies that the geometrical condition should be checked only for columns whose distillate lies on $\mathrm{ab}$ and whose bottom product lies on $\mathrm{bH}$ (i.e. columns with distillate and bottom product lying on ab are not permitted in the infinite number of trays case). If the distillate lies on $\mathrm{cb}$ then the geometrical condition is not satisfied for any bottoms product on $\mathrm{bH}$. However, if $\mathrm{D}$ lies on ac then for any $\mathrm{B}$ on $\mathrm{bH}$ the geometrical condition is satisfied. Figure 12b shows the continuation path of all possible column profiles for a given feed. The ratio $\mathrm{FB} / \mathrm{DF}$ and therefore $D$ decreases as $\mathrm{D}$ moves from a to $\mathrm{c}$ and hence multiplicities 
exist. Similarly, for the $a \rightarrow b \rightarrow I$ route, the condition for multiplicities is satisfied if $D$ lies on ac and the bottoms composition is any point on $\mathrm{Ib}$. Note that the geometrical condition is not satisfied for any $D^{\prime}$ but only for $D^{\prime}$ sufficiently close to $D$. Also note that the geometrical condition is not satisfied for the other two routes. Therefore multiplicities exist for this mixture for feed compositions which can be separated into a distillate lying on ac and a bottom product lying on $\mathrm{Ib}$ or $\mathrm{bH}$ i.e. for feeds located in the convex hull formed by ac and IH.

In the case examined above, provided $\mathrm{D}$ lies on ac, the geometrical condition is satisfied for any $\mathrm{B}$ on IH. This is due to the fact that $\mathrm{IH}$ is a straight line. The most general case where both $\mathrm{D}$ and $\mathrm{B}$ lie on curved boundaries is illustrated by Figure 13. In this figure, point $e$ is the location on ab where the tangent to the ab boundary is parallel to the tangent to the bc boundary at point $c$. Similarly, $f$ is the point on bc where the tangent to the $b c$ boundary is parallel to the tangent to the ab boundary at point a. For some $D$ on ab, there exist some $\mathrm{B}$ on bc that satisfy the geometrical condition. In general, for each $\mathrm{D}$ on ae there exists a different set $S_{B}(\mathrm{D})$ of bottoms compositions that satisfies the geometrical condition. For example if $\mathrm{D}$ is located at point a then $S_{B}(\mathrm{D})$ is the boundary segment $\mathrm{fc}$ while if $\mathrm{D}$ is located at e the $S_{B}(\mathrm{D})$ is just the point $\mathrm{c}$. Hence for each $\mathrm{D}$ the appropriate feed composition is the convex hull formed by $\mathrm{D}$ and $S_{B}(\mathrm{D})$. Therefore, the feed compositions that exhibit multiplicities lie in the union of all the convex hulls formed by $D$ and the corresponding $S_{B}(\mathrm{D})$. In Figure 13 the appropriate feed region is shaded and it is clear that it does not coincide with the convex hull formed by ae and fc.

\subsection{Summary}

In this part we examined in detail the existence of multiple steady states in the $\infty / \infty$ case of a ternary mixture. More specifically, we answered the following questions: Given a ternary mixture and its residue curve diagram,

(1) find whether multiple steady states exist for some feed composition and

(2) locate the feed composition region that lead to these multiple steady states.

The necessary and sufficient geometrical condition for the existence of multiple steady states (question 1 ) is summarized in the following:

The continuation path is defined as the path generating all possible column profiles starting from the profile with $D=0$ and ending at the profile with $D=F$. Multiple steady states occur when $D$ decreases along this path. This can be checked by the following procedure: Pick a distillate $\mathrm{D}$ and a bottom product $\mathrm{B}$, both located on some distillation region boundaries and such that the column profile that runs from $D$ to $B$ along the distillation region boundaries contains at least one saddle singular point (type III column profile). Now pick $\mathrm{D}^{\prime}$ and $B^{\prime}$ sufficiently close to $D$ and $B$ respectively and such that the column profile from $D^{\prime}$ to $B^{\prime}$ is a "later" profile along the continuation path. For the existence of multiple steady states it is required that: As we move along the continuation path from $D$ to $D^{\prime}$ and accordingly from $B$ to $B^{\prime}$, the line that passes from $D$ and is parallel to $B B^{\prime}$ crosses the $D^{\prime} B^{\prime}$ line segment.

The condition for the appropriate feed region (question 2 ) is summarized in the following: 
Pick a distillate $\mathrm{D}$. Find the set of all bottom products such that the geometrical condition is satisfied for the picked D. Name this set $S_{B}(\mathrm{D})$. For the chosen D the appropriate feed composition is the convex hull formed by $\mathrm{D}$ and $S_{B}(\mathrm{D})$. Pick another distillate and repeat. In general, for each distillate there exists a different set of bottoms compositions that satisfies the geometrical condition. Therefore, the feed compositions that lead to multiple steady states lie in the union of all the convex hulls formed by $\mathrm{D}$ and the corresponding $S_{B}(\mathrm{D})$.

\section{Finite Reflux and Finite Number of Trays}

The $\infty / \infty$ case is the limiting case of high reflux and a large number of trays. Therefore, if the geometrical condition is satisfied for a given residue curve diagram then multiplicities will exist for some sufficiently large finite reflux and finite number of trays. However, the inverse is not true. The geometrical condition is only a sufficient condition for the existence of multiplicities when the reflux and the number of trays are finite. At infinite reflux, the column profiles coincide with residue curves. This is not true at finite reflux. Moreover, column profiles at finite reflux depend on the location and the number of the feed streams. Therefore, the residue curve map cannot be used for the study of the finite reflux and finite number of trays case.

In this section, first we present steady state bifurcation results for the mixture acetone (L) - heptane (H) - benzene (I-E) which show that the prediction for the existence of multiple steady states in the $\infty / \infty$ case carries over to columns operating at finite reflux and with a finite number of trays. We further show that, although the predictions were made in the $\infty / \infty$ case, it does not mean that multiple steady states do not exist for realistic operating conditions (low reflux and number of trays). However, apart from the fact that the $\infty / \infty$ case predictions carry over, the results presented here should not be generalized because they are specific to the particular example. The column characteristics are depicted in Figure 14. In this column, a mixture of $90 \%$ acetone and $10 \%$ heptane (the azeotropic composition is $93 \%$ acetone and $7 \%$ heptane) is separated using benzene as the entrainer. Acetone is recovered in the distillate while the bottom product (heptane and benzene) is fed to the entrainer recovery column (Figure 2) from which heptane is recovered and benzene is recycled to the azeotropic column. For this example, the distillate, reflux and entrainer flows as well as the number of stages are treated as parameters. The bifurcation calculations were conducted with AUTO, a software package developed by Doedel (1986).

\subsection{Varying the Distillate Flow}

Figure 15 shows typical bifurcation diagrams with the distillate flow as the bifurcation parameter for the column depicted in Figure 14 with $E / F=1$ and various $R$. If $R$ is low enough $(R / F=2)$, a unique steady state is calculated by the continuation algorithm. For higher values of $R(R / F=4,10,50)$, multiple steady states exist for some $D$. In these cases, a unique stable steady state exists for low $D$. $D$ increases until the continuation algorithm reaches the first limit point. Beyond that point an unstable steady state is calculated (dashed curve). Along this part of the continuation path $x_{D L}$ increases while $D$ decreases until the second limit point is encountered. Beyond the second limit point, $D$ increases again and a second stable steady state is calculated. Hence, two stable and one unstable steady states 
exist for distillate flows between the two limit points (multiplicity region); a unique stable steady state exists otherwise.

Note the similarity of those continuation paths with the continuation path we tracked in the $\infty / \infty$ case (Figure 6 ). Also note that in Figure 15 the multiplicity region expands as the reflux flow increases. Figures $16 \mathrm{a}$ and $16 \mathrm{~b}$ show the reflux - distillate multiplicity region for two different entrainer feed flows. As the reflux decreases, the multiplicity region becomes more narrow and at some point the multiplicities vanish. Note that, although those multiple steady states were predicted at infinite reflux, they still exist at very low reflux values. Note also that, since the overall feed does not lie on the line connecting the azeotrope with the pure benzene (I) corner, the distillate flow multiplicity interval of this column in the $\infty / \infty$ case is not between $L$ and $L+H(90$ and $100 \mathrm{kmol} / \mathrm{min})$ but between 90 and $96.6(=90 / 0.93)$ $\mathrm{kmol} / \mathrm{min}$. Moreover, the column has only 4 trays in the lower section and therefore some discrepancy from the $\infty / \infty$ case prediction is expected. In addition, note that the column with $E=1 \mathrm{kmol} / \mathrm{min}$ is much closer to the infinite relfux and infinite reboil condition than the column with $E=200 \mathrm{kmol} / \mathrm{min}$. The above explains why the distillate multiplicity region at high reflux in Figure $16 \mathrm{a}(E=1 \mathrm{kmol} / \mathrm{min})$ is much closer to the $\infty / \infty$ case prediction than the corresponding region of Figure $16 \mathrm{~b}(E=200 \mathrm{kmol} / \mathrm{min})$.

\subsection{Varying the Entrainer and Reflux Flows}

In these studies, the column depicted in Figure 14 is used with the distillate flow fixed at $90.9 \mathrm{kmol} / \mathrm{min}$. The bifurcation calculation results are summarized in Figure 17 . The four pictures at the bottom of Figure 17 show typical bifurcation diagrams with the entrainer feed flow as the bifurcation parameter for various fixed reflux flows. At very low reflux, a unique stable steady state exists for all entrainer feed flows. As the reflux increases, three multiple steady states appear for some entrainer feed flow interval. Like in the case where the distillate flow is the bifurcation parameter, there is just one continuation path with two limit points. For entrainer flows between the two limit points, three steady states exist. The dashed curve depicts the unstable steady state. The entrainer flow range between the two limit points expands as reflux increases. Figure 18 shows the actual bifurcation diagram for $R=500 \mathrm{kmol} / \mathrm{min}$.

The six pictures on the right side of Figure 17 show typical bifurcation diagrams with the reflux flow as the bifurcation parameter for various entrainer flows. Contrary to the cases where the entrainer and the distillate flows are the bifurcation parameters, there are generally two separate continuation paths in each diagram. One of them expands along the whole range of reflux from zero to infinity. Along this path, a stable steady state is calculated. The second path generally extends to infinite reflux but vanishes at some finite reflux flow (limit or turning point). Along this second path, one stable and one unstable steady state are calculated.

At high entrainer flows, the second (two-steady-state) path lies below the single steady state path while the situation is reversed at low entrainer flows. Therefore, at high entrainer flows the unstable state is "connected" to the low conversion stable state whereas at low entrainer flows it is "connected" to the high conversion stable state. Hence, three steady states exist for reflux flows above the limit point while a unique stable steady state exists for reflux flows below that limit point. Moreover, as the entrainer flow is decreased the limit 
point of the two steady state path moves to lower reflux values. The above characteristics can be seen in the upper four pictures on the right of Figure 17. Figure 19 shows the actual bifurcation diagram for $E=80 \mathrm{kmol} / \mathrm{min}$.

At very low entrainer flows, the two-steady-state path does not extend to infinite reflux and a second limit point appears at high reflux. At even lower entrainer flows, the two steady state path disappears and a unique steady state exists throughout. The above are illustrated by the two lower pictures on the right of Figure 17.

Finally, the central picture of Figure 17 shows the entrainer-reflux multiplicity region. The multilpicity region expands as reflux is increased. Note that multiplicities persist for low entrainer and reflux flows which is the region of operation in practice.

\subsection{Effect of the number of trays}

In the first part of this article we have shown that multiplicities exist for columns with an infinite number of trays. Doherty and Perkins (1982) proved that multiplicities cannot exist for single-staged "columns." It is expected then, that multiplicities vanish as the number of trays decreases below some critical number.

The effect of decreasing the number of stages is depicted via bifurcation diagrams where the distillate and reflux flows are fixed and the entrainer flow is the bifurcation parameter. Figure 20 shows four such diagrams for columns similar to the ones depicted in Figure 14 i.e. with the feed location fixed on tray 4 and different number of stages $N$. Three steady states exist for some very narrow entrainer flow interval for the columns with 23 and 22 trays while multiplicities vanish for the 21 and 15 tray columns. Figure 21 shows the entrainer - reflux multiplicity region for three columns with 44, 33 and 23 stages and fixed distillate flow. It is apparent that the multiplicity region for the 23 tray column is very narrow. Moreover, no multiplicities were found for columns with less than 22 trays. Therefore, multiplicities vanish for columns with number of stages below some critical value. However, the relationship between the number of stages and the location of the multiplicity region in the entrainer - reflux plane is not clear.

\subsection{Curved Boundaries}

In this subsection we present an example which illustrates that highly curved boundaries can induce multiplicities. The ternary mixture under consideration is that of acetone (L), methanol (H) and chloroform (I) also studied by Kienle et al. (1992). The corresponding residue curve diagram is shown in Figure 22a. It belongs to the 311-s residue curve diagram class. There are three binary azeotropes $(a, b, c)$ and one saddle ternary azeotrope $(s)$ in this diagram.

The interior residue curve boundaries (Figure $22 \mathrm{~b}$ ) divide the composition triangle in four distillation regions and therefore there are eight routes (two for each region) from an unstable node to a stable node along the distillation region boundaries. Note that none of the routes contains two neighboring saddles and that sa is the only boundary that is highly curved and therefore might induce multiplicities.

There exist two routes that contain sa, namely $c \rightarrow s \rightarrow a$ and $b \rightarrow s \rightarrow a$. Now check the geometrical condition by tracking each route in the proper direction (the proper direction is 
the direction of the continuation path i.e. the one that starts with the distillate located at the unstable node and ends with the bottoms located at the stable node). It is very simple to show that the distillate flowrate increases monotonically for the $c \rightarrow s \rightarrow a$ route. In contrast, the geometrical condition is satisfied for some feed locations as we track the $b \rightarrow s \rightarrow a$ route. The shaded region in Figure 22b depicts the appropriate feed composition region for which multiplicities will be observed in the $\infty / \infty$ case.

The above findings are supported by simulation results for a column with 30 trays, $D / F=.5, R / F=100$, a feed composition of $26.5 \%$ acetone, $23 \%$ methanol and $50.5 \%$ chloroform , a feed flowrate of $100 \mathrm{kmol} / \mathrm{min}$ and a feed tray located at stage 14. Figures 23a-c show the three different column profiles (two stable and one unstable) with the above specifications. Figure 23d shows the location of the three profiles relative to the distillation region boundaries in the composition triangle. Note that the column operates at high reflux but not at infinite reflux and does not have an infinite number of trays. Hence, it is expected that the column profiles do not exactly follow the residue curve boundaries.

Finally, Figure 24 shows bifurcation diagrams with the distillate flow as the bifurcation parameter for $R / F=1,5$ and 100 . The three multiple steady states persist for reflux to feed ratios as low as 1 .

\section{Effect of the VLE model}

The geometrical sufficient condition for the existence of multiplicities at finite reflux is based on arguments about the distillation region boundaries at infinite reflux. In general, the orientation and the curvature of those boundaries depends on the specific thermodynamic model used. Therefore it is expected that switching from one VLE model to another may affect the existence of multiplicities

- quantitatively only i.e. multiplicities still exist but there are different appropriate feed composition regions, distillate - reflux multiplicity regions etc.

- qualitatively i.e. multiple steady states exist using one model while they do not exist using another one.

For example, in the acetone - heptane - benzene case (001 class) multiple steady states exist in the $\infty / \infty$ case for any feed composition inside the composition triangle, regardless of the specific thermodynamic model used. This independence from the thermodynamic model is inherent in any 001 class residue curve diagram. However, quantitative differences between the models exist for the entrainer - reflux and distillate - reflux multiplicity regions. On the other hand, when the existence of multiplicities depends on the orientation and/or the curvature of some interior residue curve boundaries, it is possible that multiple steady states exist when using one thermodynamic model while they do not exist when using another model.

Finally, a note on the fact that the geometrical condition is not necessary for the existence of multiple steady states in the finite reflux case. Finite reflux boundaries are not as rigorously defined as infinite reflux ones. It is known that the shape of the distillation boundaries changes with reflux. Therefore, it may be possible that multiplicities exist at 
some range of finite reflux flows (due to some "distorted" boundaries) while the geometrical condition is not satisfied for infinite reflux boundaries. The above can cause another discrepancy observed for different thermodynamic models.

\section{Effect on Design and Operation}

In this section we briefly discuss the effect of multiplicities on the distillation column design and operation. First we examine the problem of avoiding the multiplicity region (i.e operating in the single steady state region) and meeting the product specifications (defined later). Here, the number of stages and the entrainer flow are fixed while the distillate and reflux are the design parameters. The column specifications are $99 \%$ purity of acetone in the distillate and $99 \%$ acetone recovery. By superimposing the reflux-distillate regions where the above

specifications are met on the corresponding multiplicity regions (Figures $16 \mathrm{a}$ and 16b) we obtain Figures 25a and $25 \mathrm{~b}$ for the two fixed entrainer flows. If $E=1 \mathrm{kmol} / \mathrm{min}$ (Figure 25a) the column specifications are only met inside the multiplicity region and therefore multiplicities cannot be avoided in this case. However, if the entrainer flow is increased to $200 \mathrm{kmol} / \mathrm{min}$, there exists a reflux-distillate region where the specifications are met and a unique steady state exists. Therefore, we can meet the column specifications and avoid the multiplicity region at the expense of a higher entrainer feed flow.

Finally, we examine whether it is possible to "jump" from the high conversion stable steady state to the corresponding low conversion stable steady state under a feed composition disturbance while operating in the multiplicity region. In some sense, we examine whether it is necessary to operate in the single steady state region. Here, the column has 44 trays, the entrainer flow is $1 \mathrm{kmol} / \mathrm{min}$, the reflux flow is $1000 \mathrm{kmol} / \mathrm{min}$ and no control action is used (open-loop behavior). The feed originally contains $90 \%$ acetone and $10 \%$ heptane and under these conditions three steady states exist. The column originally operates at the high conversion state ( $99 \%$ acetone in distillate). From time zero to 6000 seconds the feed composition is changed to $91 \%$ acetone and $9 \%$ heptane. Note that under these conditions a single (low conversion) stable steady state exists. Finally, at time 6000 seconds the feed composition is changed back to its original value. Figure 26 shows that the column profile "jumps" from the high conversion state to the low conversion state $(93.17 \%$ acetone in distillate) because of the feed composition disturbance. The calculations were repeated for smaller disturbance time intervals $(1000,2000,4000$ seconds) but this time the acetone composition returned to its original $99 \%$ purity. Therefore, it seems that for this particular design it is relatively difficult to "jump" from one stable profile to the other and hence, this result disputes whether it is necessary to operate in the single steady state region.

However, the material presented in this section is just a brief illustration of the implications of multiplicities on column design and operation and a more thorough investigation of this subject is needed.

\section{Conclusions}

In this article we study multiple steady states in ternary homogeneous azeotropic distillation. First we examine in detail the infinite reflux and infinite number of trays $(\infty / \infty)$ case. We 
present a systematic procedure which determines whether multiplicities exist for any given residue curve diagram and feed composition. Through this procedure we answered the following questions:

Given a ternary mixture and its residue curve diagram, we can for the $\infty / \infty$ case

(1) find whether multiple steady states exist for some feed composition and

(2) locate the region of feed compositions that lead to these multiple steady states.

We derive (1) the necessary and sufficient geometrical condition for the existence of multiple steady states and (2) the condition the feed compositions must satisfy to lead to multiple steady states. A few other important results are the following:

In the case of straight boundaries we found that two neighboring saddles is a necessary condition for the existence of mutliplicities.

If multiple steady states exist under the straight boundaries assumption, then, assuming that the azeotropic compositions do not change, these multiplicities still exist even if the boundaries are curved, although the appropriate feed region is changed.

Highly curved boundaries (pseudosaddles) can induce multiple steady states.

For columns operating at finite reflux the geometrical condition is only a sufficient condition for the existence of multiple steady states. We use an example to show that the prediction for the existence of multiple steady states in the $\infty / \infty$ case carries over to columns operating at finite reflux and with a finite number of trays. We further show that, although the predictions were made in the $\infty / \infty$ case, it does not mean that multiple steady states do not exist for realistic operating conditions (low reflux and entrainer feed flows and small number of trays). However, apart from the fact that the $\infty / \infty$ case predictions carry over, the observations presented here should not be generalized because they are specific to the particular example. We also present an example which illustrates that highly curved boundaries can induce multiplicities.

We offered some comments on the effect of the thermodynamic model on the existence of multiplicities and we show that some of the results presented here do not depend on the specific thermodynamic model used. Finally, we briefly discuss the effect of multiplicities on the column design and operation. The consideration here is whether it is necessary to operate in the single steady state region (i.e. avoid the multiplicity region). A more thorough investigation of this topic is needed.

Acknowledgements: We acknowledge gratefully the financial support of the Donors of the Petroleum Research Fund administered by the American Chemical Society and of the I. S. Latsis Foundation. We also thank Prof. Skogestad (Norwegian Technical University, Trondheim) for several enlightened discussions. Finally, we thank Prof. Doherty and Jeffrey Knapp (University of Massachusetts, Amherst) for providing us thermodynamic data and subroutines. 


\section{Literature Cited}

Chavez, R. C., J. D. Seader and T. L. Wayburn, "Multiple Steady-State Solutions for Interlinked Separation Systems," Ind. Eng. Chem. Fund., 1986, 25, pp. 566-576.

Doedel, E., "AUTO: Software for Continuation and Bifurcation Problems in Ordinary Differential Equations," Applied Mathematics, Caltech, Pasadena, CA (1986).

Doherty, M. F., and J. D. Perkins, "On the Dynamics of Distillation Processes. I. The Simple Distillation of Multicomponent Non-reacting, Homogeneous Liquid Mixtures," Chem. Eng. Science, 1978, 33, pp. 569-578.

Doherty, M. F., and J. D. Perkins, "On the Dynamics of Distillation Processes. IV. Uniqueness and Stability of the Steady State in Homogeneous Continuous Distillation," Chem. Eng. Science, 1982, 37, pp. 381.

Jacobsen, E. W., and S. Skogestad, "Multiple Steady States in Ideal Two-Product Distillation," AIChE Journal, 1991, 37(4), pp. 499-511.

Kienle, A., W. Marquardt, and E. D. Gilles, "Steady State Multiplicities in Homogeneous Azeotropic Distillation Processes," AIChE Annual Meeting, Miami, 1992.

Kocach III, J. W., and W. D. Seider, "Heterogeneous Azeotropic Distillation - HomotopyContinuation Methods," Comput. Chem. Eng., 1987, 11(6), pp. 593-605.

Laroche, L., N. Bekiaris, H. W. Andersen, and M. Morari, "The Curious Behavior of Homogeneous Azeotropic Distillation - Implications for Entrainer Selection," presented at the AIChE Annual Meeting, Chicago, 1990.

Laroche, L.,"Homogeneous Azeotropic Distillation: Entrainer Selection", Ph. D. Dissertation, California Institute of Technology, Pasadena, 1991.

Laroche, L., N. Bekiaris, H. W. Andersen, and M. Morari, "Homogeneous Azeotropic Distillation: Separability and Flowsheet Synthesis," Ind. Eng. Chem. Res., 1992, 31(9), pp. 2190-2209.

Lin, W. J., J. D. Seader and T. L. Wayburn, "Computing Multiple Solutions to Systems of Interlinked Separation Columns," AIChE Journal, 1987, 33(6), pp. 886-897.

Magnussen, T., M. L. Michelsen, and Aa. Fredenslund, "Azeotropic Distillation Using UNIFAC," Inst. Chem. Eng. Symp. Ser., 1979, 56, pp. 4.2/1-4.2/19.

Matsuyama, H., and H. Nishimura, "Topological and Thermodynamic Classification of Ternary Vapor-Liquid Equilibria," J. Chem. Eng. Jpn., 1977, 10(3), pp. 181-187.

Petlyuk, F. B., and V. S. Avetyan, "Investigation of three component distillation at infinite reflux," Theoretical Foundations of Chemical Engineering (in Russian), 1971, 5(4), pp. 499-506.

Prokopakis, G. J., and W. D. Seider, "Feasible Specifications in Azeotropic Distillation," AIChE Journal, 1983, 29(1), pp. 49-60.

Rosenbrock, H. H., "A Lyapunov Function with Applications to some Nonlinear Physical Systems," Automatica, 1962, 1, pp. 31-53.

Sridhar, L. N., and A. Lucia, "Analysis and Algorithms for Multistage Separation Processes," Ind. Eng. Chem. Res., 1989, 28, pp. 793-803.

Widagdo, S., W. D. Seider, and D. H. Sebastian, "Bifurcation Analysis in Heterogeneous Azeotropic Distillation," AIChE Journal, 1989, 35(9), pp. 1457-1464. 


\section{Appendix}

The appendix contains information on the thermodynamic model used in the simulations presented in this article. Vapor - liquid equilibrium calculations are based on the following equation:

$$
y_{i} P=x_{i} P_{i}^{s a t}(T) \gamma_{i}(T, \underline{x})
$$

where $\mathrm{P}=1 \mathrm{~atm}$ in all simulations presented here.

Vapor pressures were computed by the Antoine equation:

$$
\ln P_{i}^{s a t}=A_{i}+\frac{B_{i}}{T+C_{i}}
$$

where $T$ in ${ }^{\circ} K$ and $P_{i}^{s a t}$ in $N / m^{2}$. Table A1 contains the Antoine coefficients for the components used in the simulations.

Liquid activity coefficients were computed by the Van Laar equation:

$$
T \ln \gamma_{k}=\sum_{i} A_{k i} z_{i}-\sum_{i} A_{k i} z_{k} z_{i}-\sum_{j \neq k} \sum_{i \neq k} A_{j i} \frac{A_{k j}}{A_{j k}} z_{j} z_{i}
$$

where $z_{i}$ is the effective volume fraction,

$$
z_{i}=x_{i} /\left(\sum_{j} x_{j} \frac{A_{j i}}{A i j}\right) .
$$

In this model $A_{i i}=0, A_{i j}=0$ implies ideality, and if $A_{j i} / A_{i j}=0 / 0$ set $A_{j i} / A_{i j}=1$. Tables $\mathrm{A} 2$ and $\mathrm{A} 3$ contain the Van Laar coefficients for the mixtures acetone - heptane - benzene and acetone - methanol - chloroform respectively. 


\section{Table 1: Acetone concentration in the distillate for various reflux flows}

\begin{tabular}{|c|c|c|}
\hline$R(\mathrm{kmol} / \mathrm{min})$ & First stable profile & Second stable profile \\
\hline 500 & 0.93 & -- \\
\hline 1000 & 0.93 & -- \\
\hline 5000 & 0.93 & --- \\
\hline 6500 & 0.93 & -- \\
\hline 6600 & 0.93 & 0.99 \\
\hline 7000 & 0.93 & 0.99 \\
\hline 8000 & 0.93 & 0.99 \\
\hline 10000 & 0.93 & 0.99 \\
\hline 20000 & 0.93 & 0.99 \\
\hline 100000 & 0.93 & 0.99 \\
\hline
\end{tabular}




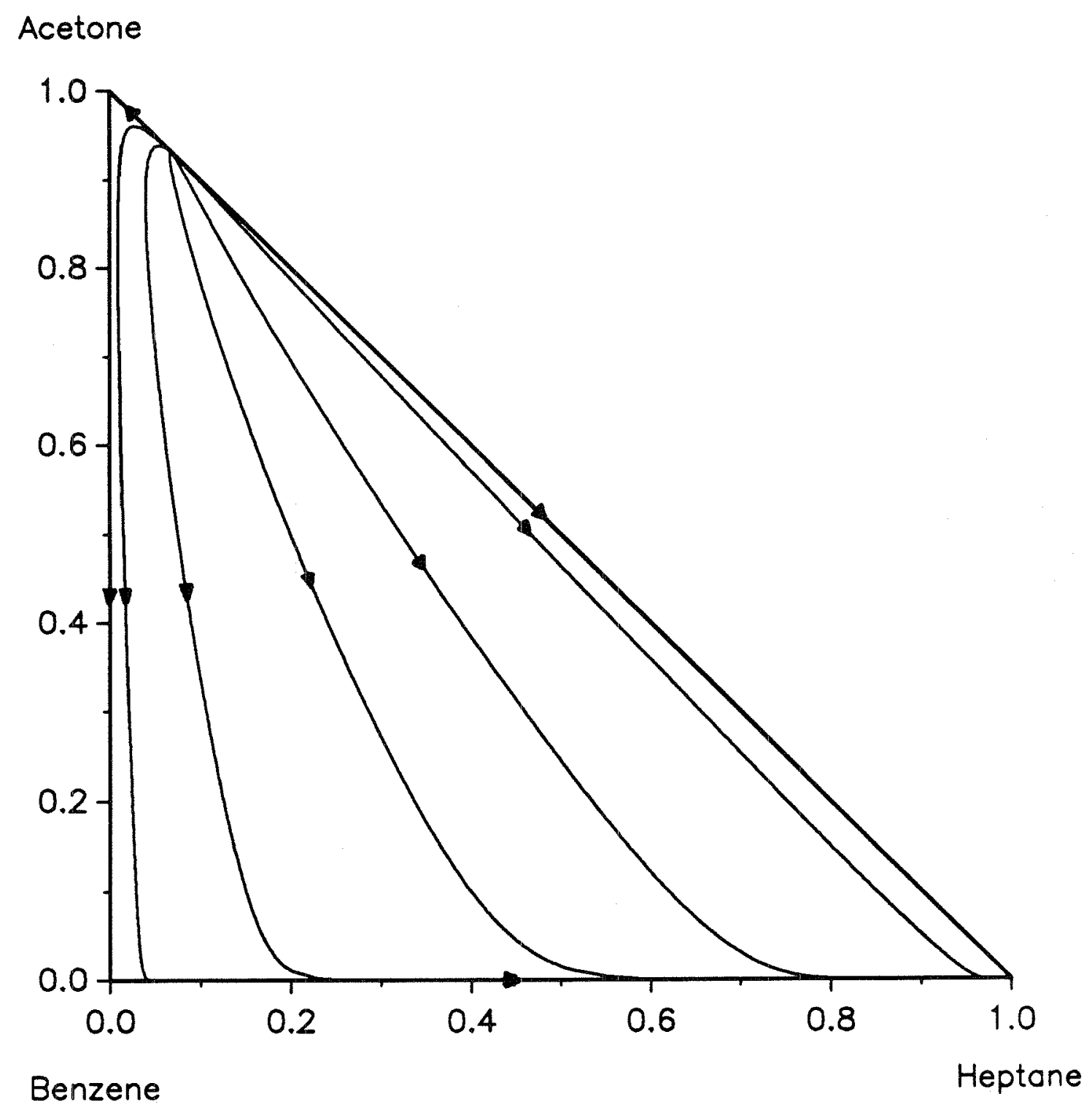

Figure 1: The acetone - heptane - benzene residue curve diagram. 


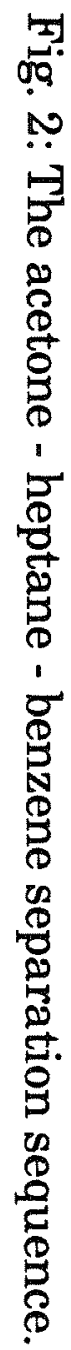
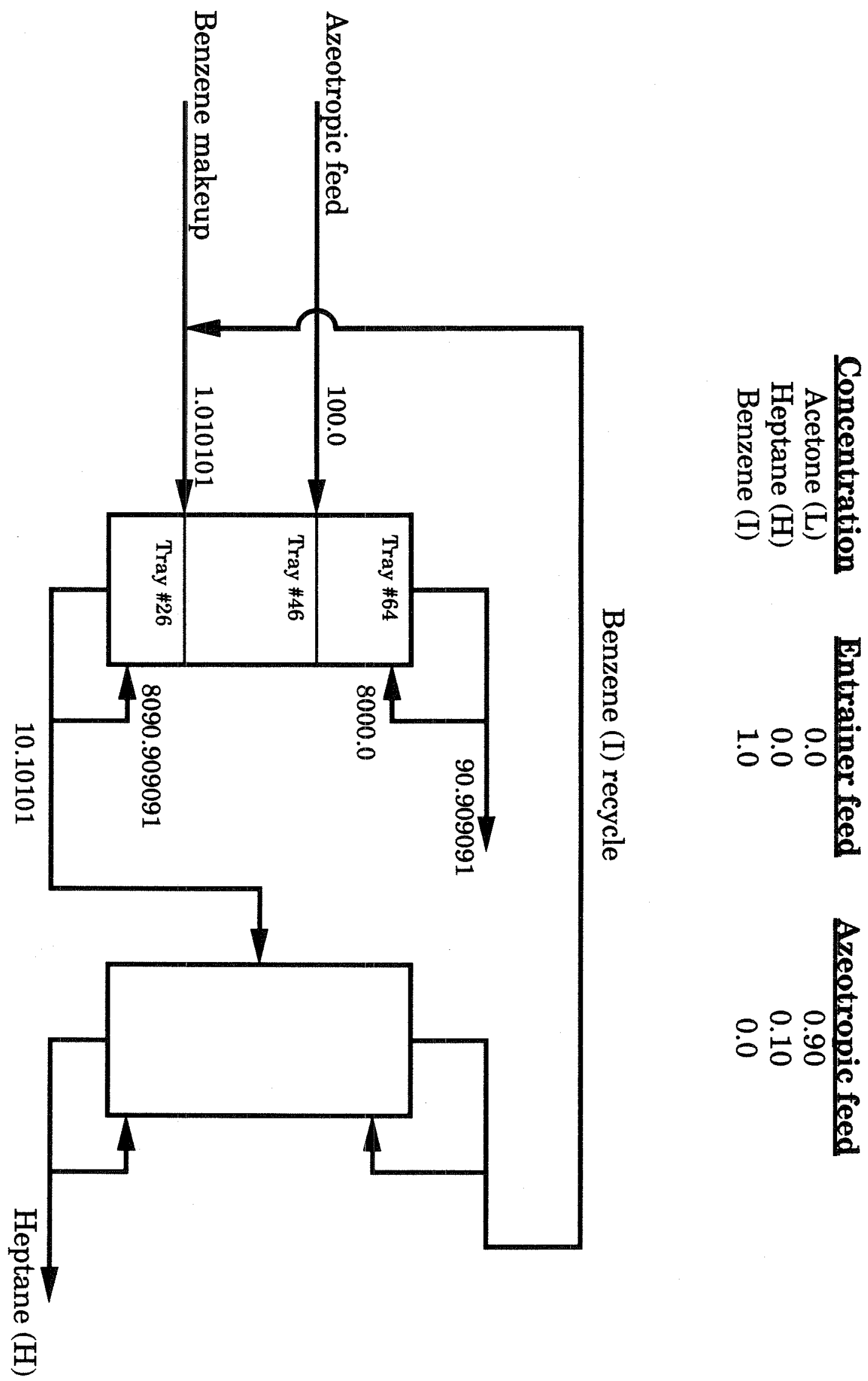


\begin{tabular}{ccc} 
Concentration & Distillate & Bottoms \\
\hline Acetone (L) & 0.99 & 0.0 \\
Heptane (H) & 0.0043 & 0.9513 \\
Benzene (I) & 0.0057 & 0.0487
\end{tabular}

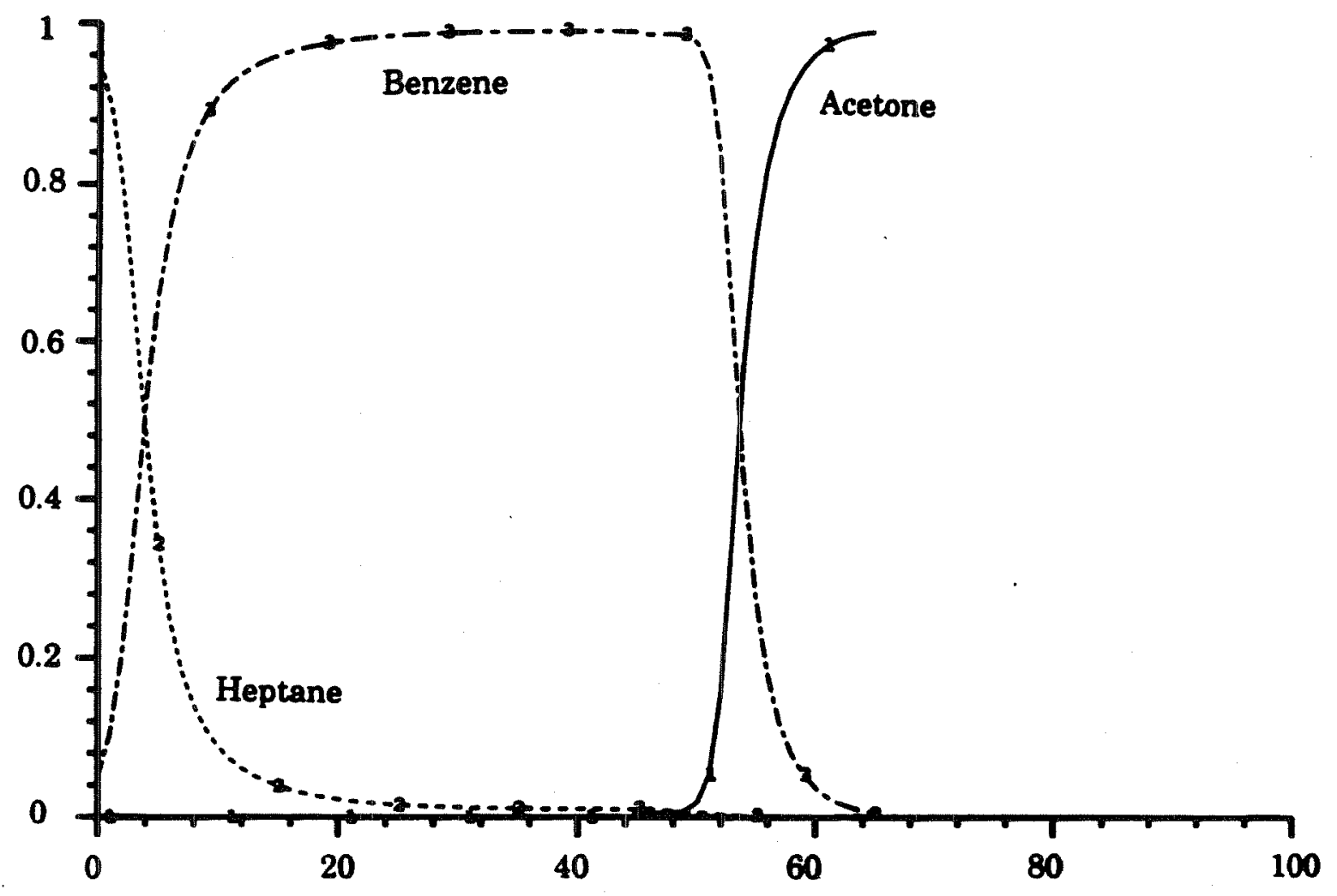

Fig. 3a: Acetone (1) - heptane (2) - benzene (3) column composition profile. High conversion steady-state. 


\begin{tabular}{ccc} 
Concentration & Distillate & Bottoms \\
\hline Acetone (L) & 0.9317 & 0.5247 \\
Heptane (H) & 0.0683 & 0.3753 \\
Benzene (I) & 0.0 & 0.1000
\end{tabular}

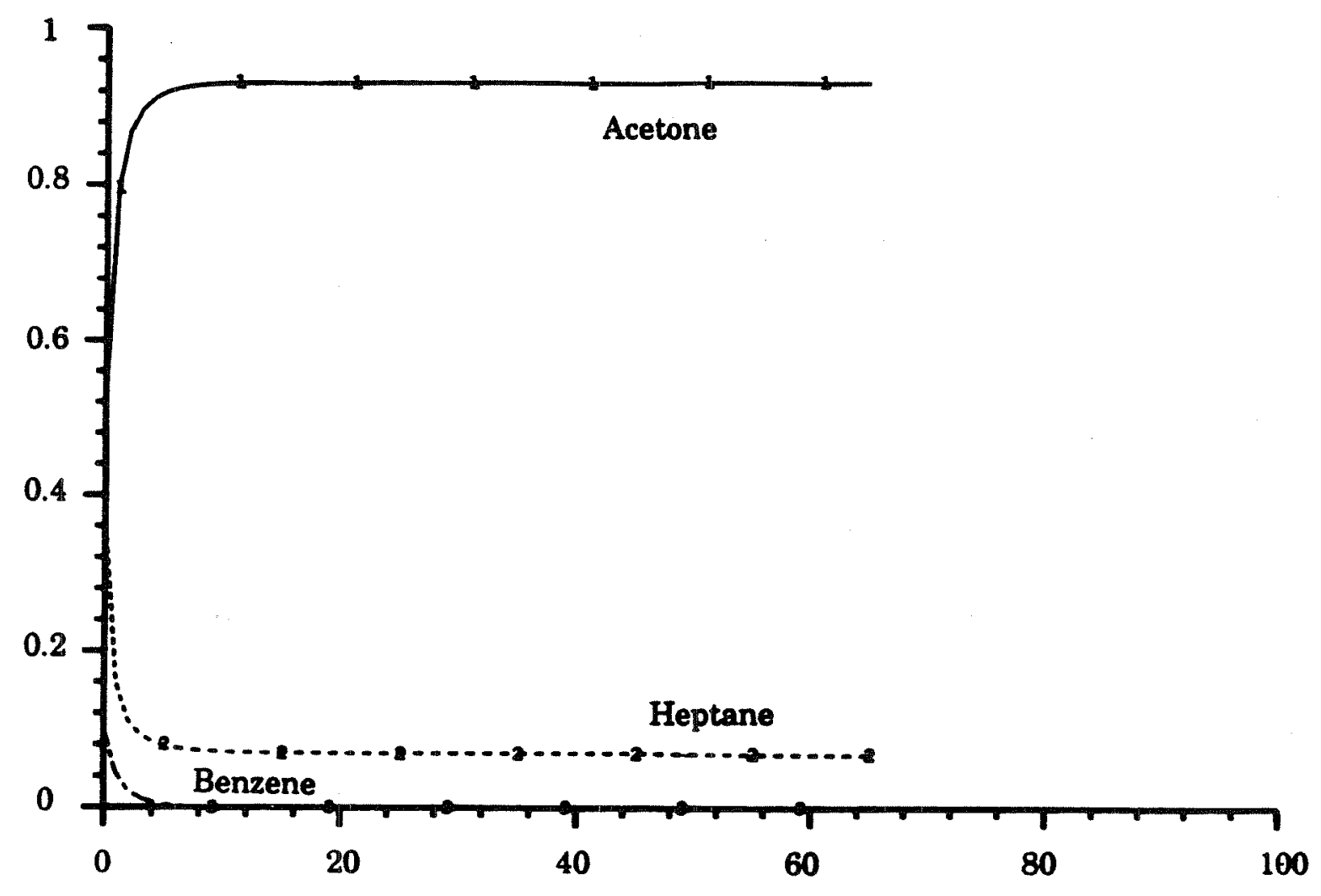

Fig. 3b: Acetone (1) - heptane (2) - benzene (3) column composition profile. Low conversion steady-state. 


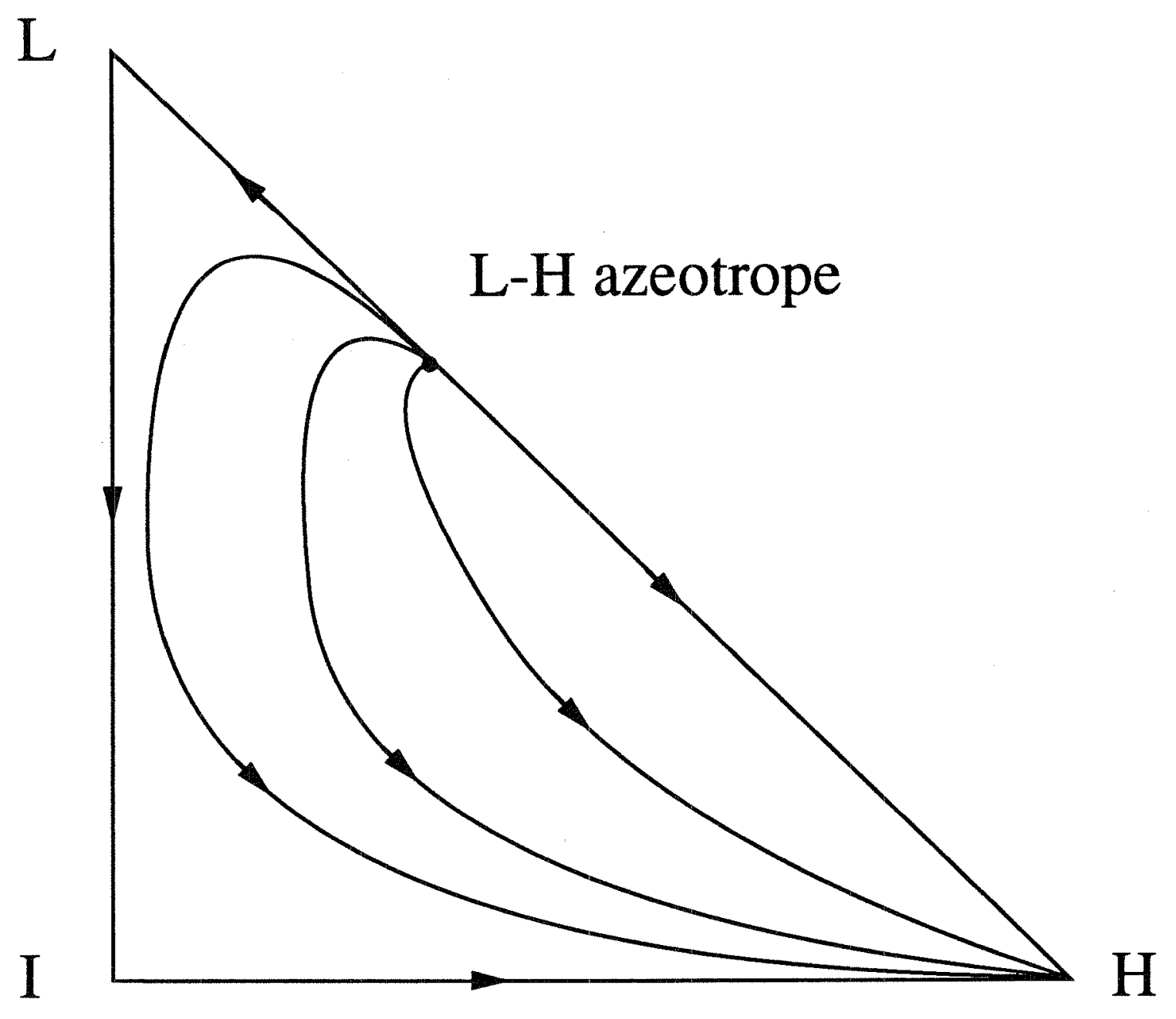

Figure 4: Residue curve diagram of a 001 class ternary mixture. 


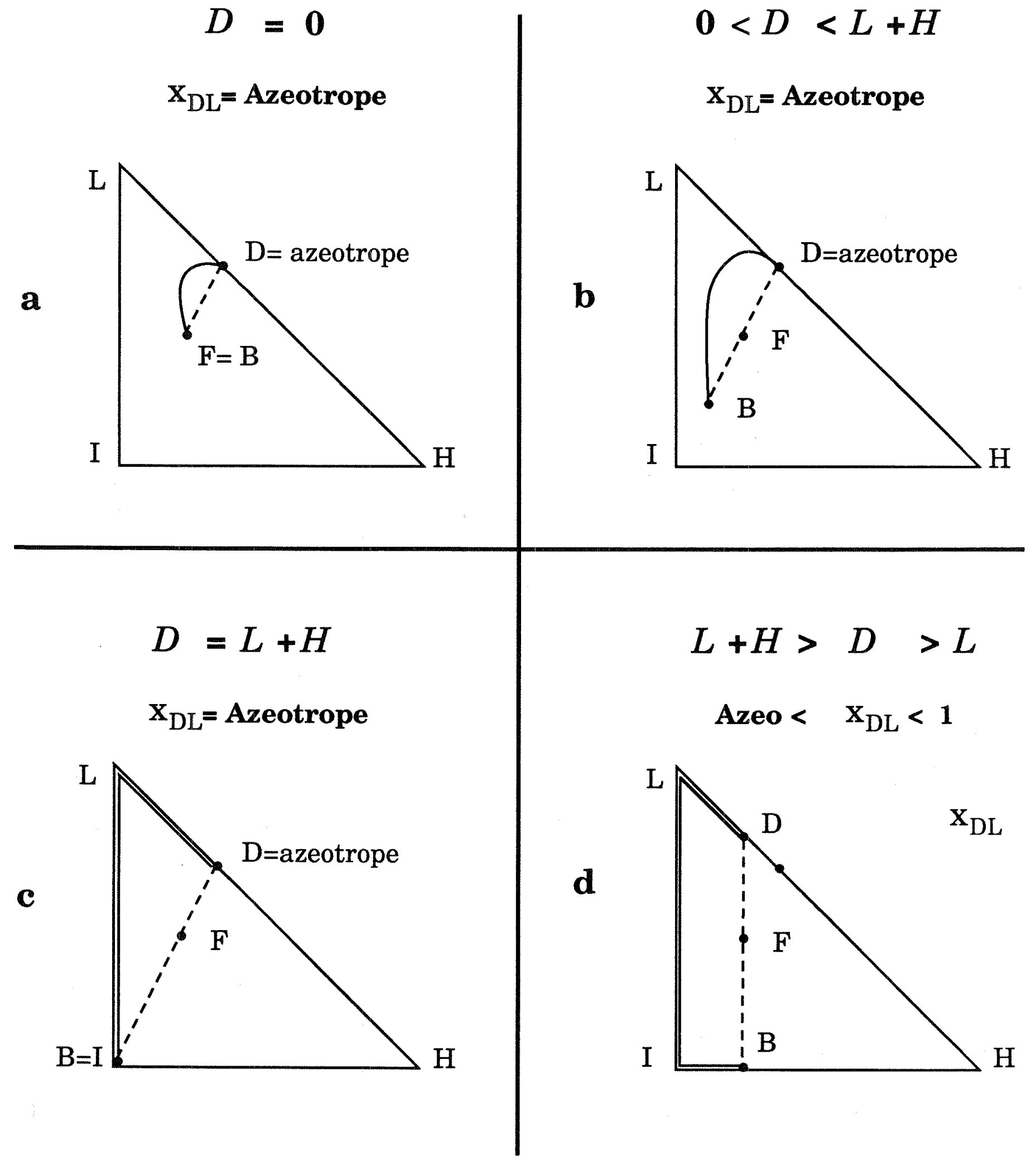

Figures 5a-5d: Column profiles with infinite number of trays at infinite reflux. 


$$
\begin{aligned}
& D=L \\
& \mathrm{x}_{\mathrm{DL}}=\mathbf{1}
\end{aligned}
$$

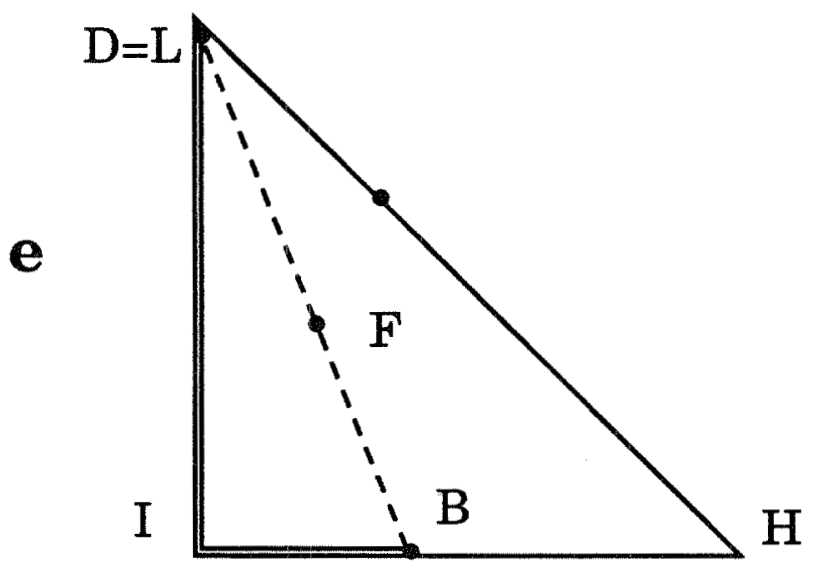

$$
L<D<L+I
$$

$1>\mathrm{x}_{\mathrm{DL}}>\frac{L}{L+I}$

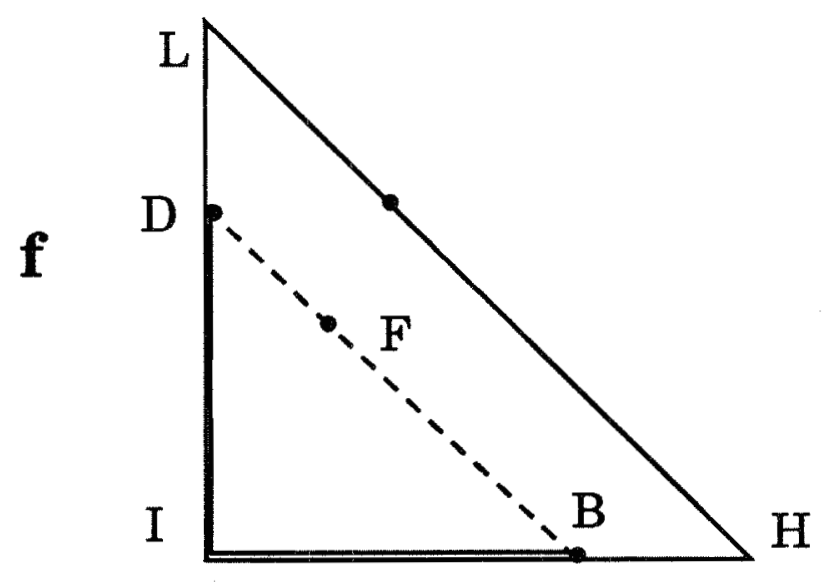

$D=L+I$

$\mathrm{x}_{\mathrm{DL}}=\frac{L}{L+I}$

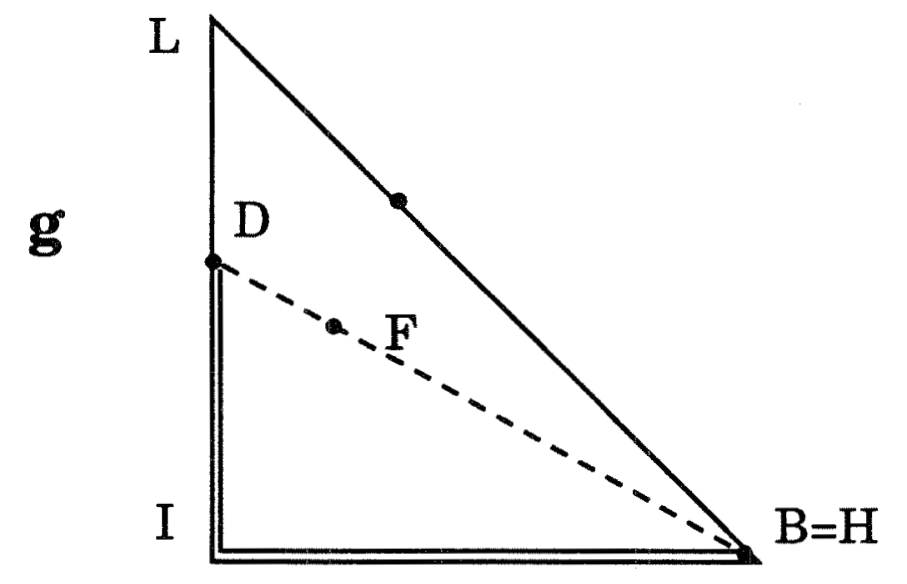

$L+I<D<L+I+H$
$\frac{L}{L+I}>\mathrm{x}_{\mathrm{DL}}>\frac{L}{L+I+H}$

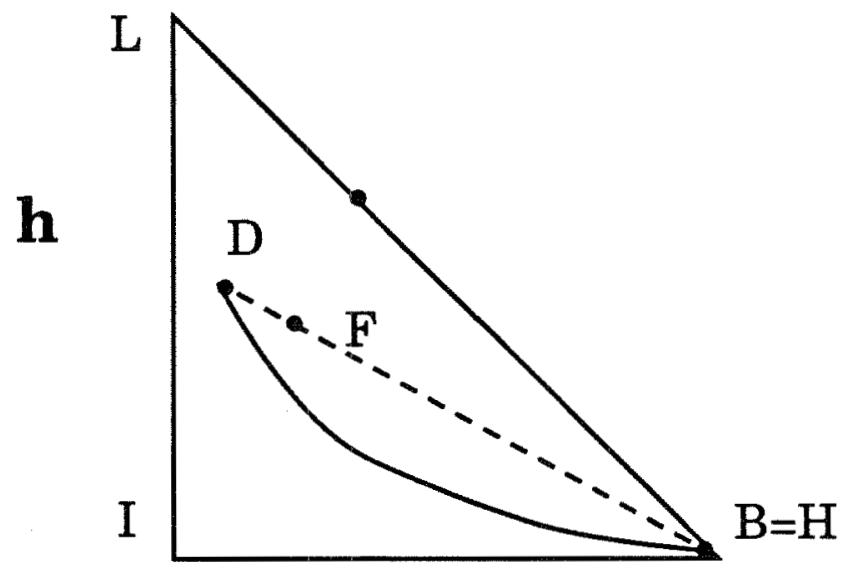

Figures 5e-5h: Column profiles with infinite number of trays at infinite reflux. 
Three Steady States exist for

$$
L+H>D>L
$$

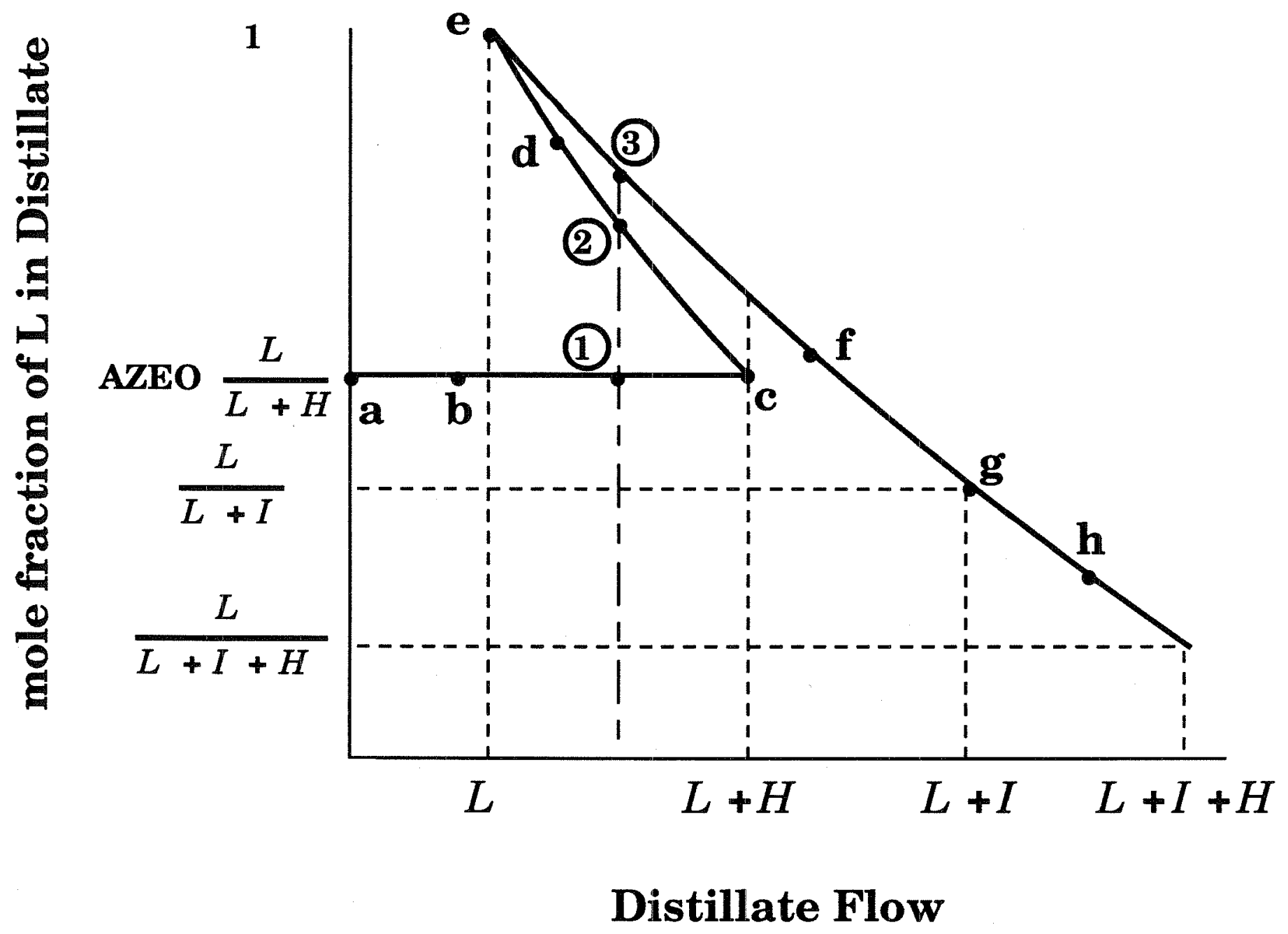

Figure 6: Composition of $L$ in the Distillate along the continuation path. 


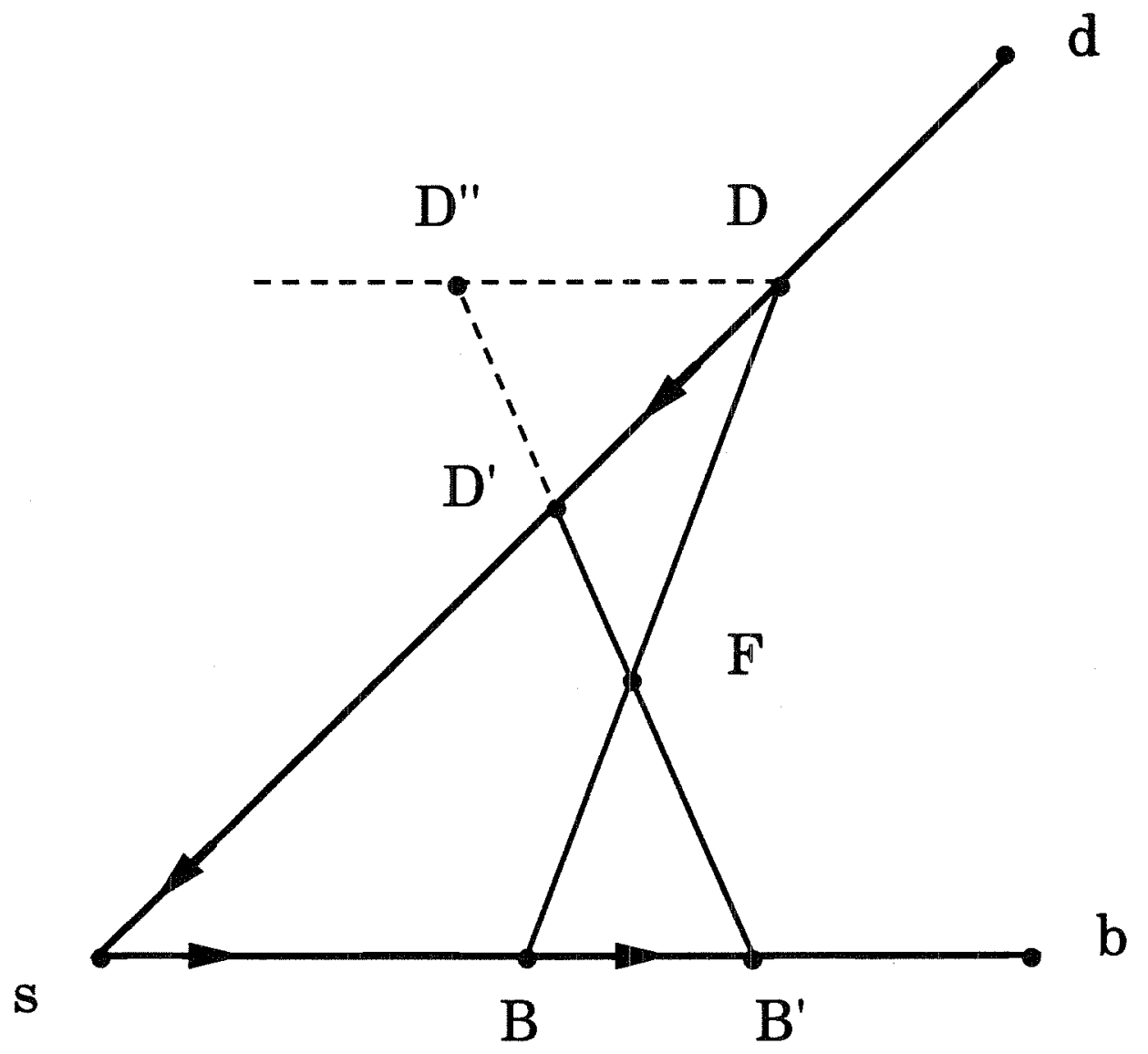

Figure 7: $D$ increases monotonically for column profiles that contain only one saddle singular point. 
$\mathbf{a}$

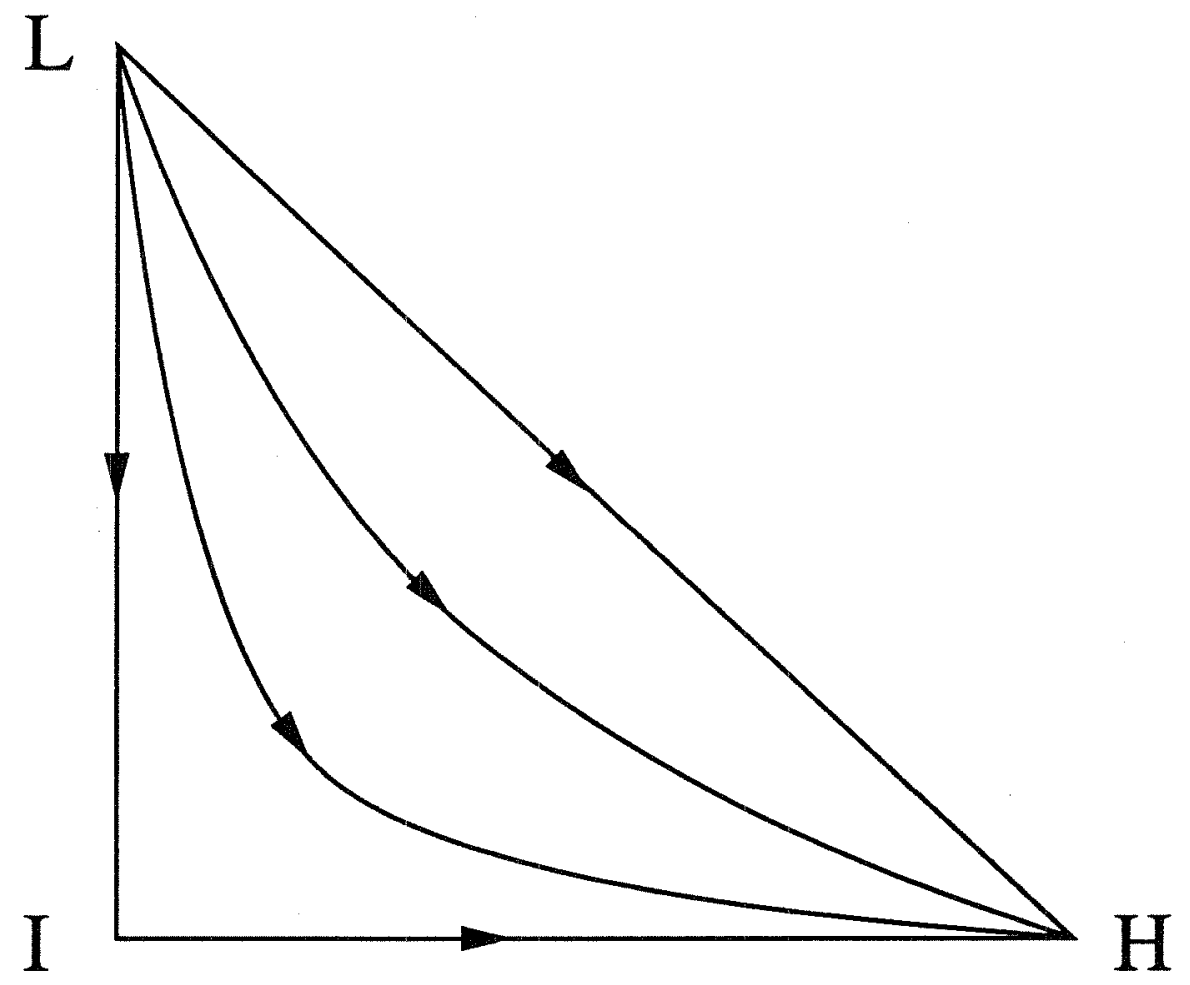

b

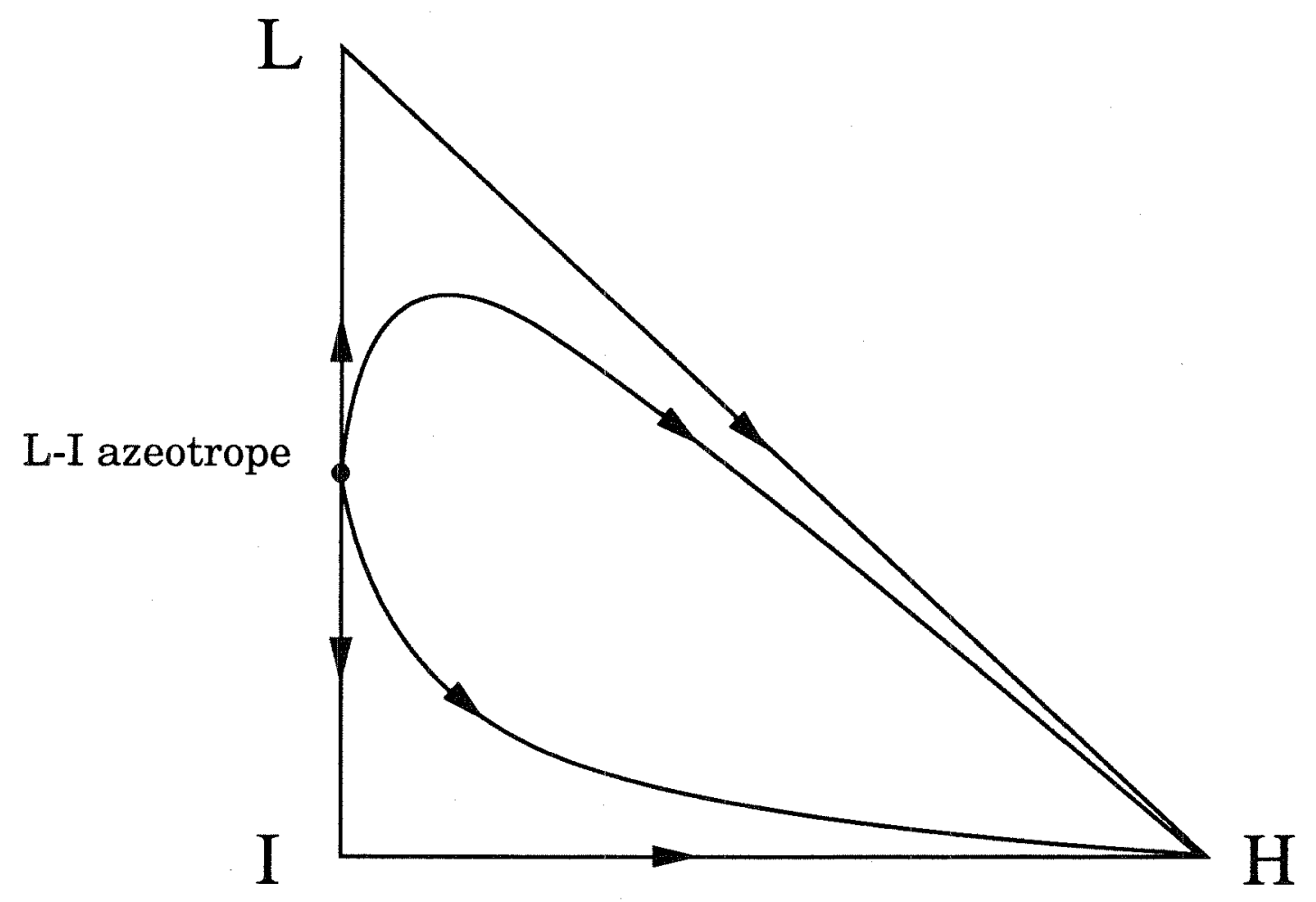

Figure 8: Residue curve diagrams of a. a 000 class b. a 100 class ternary mixture. 

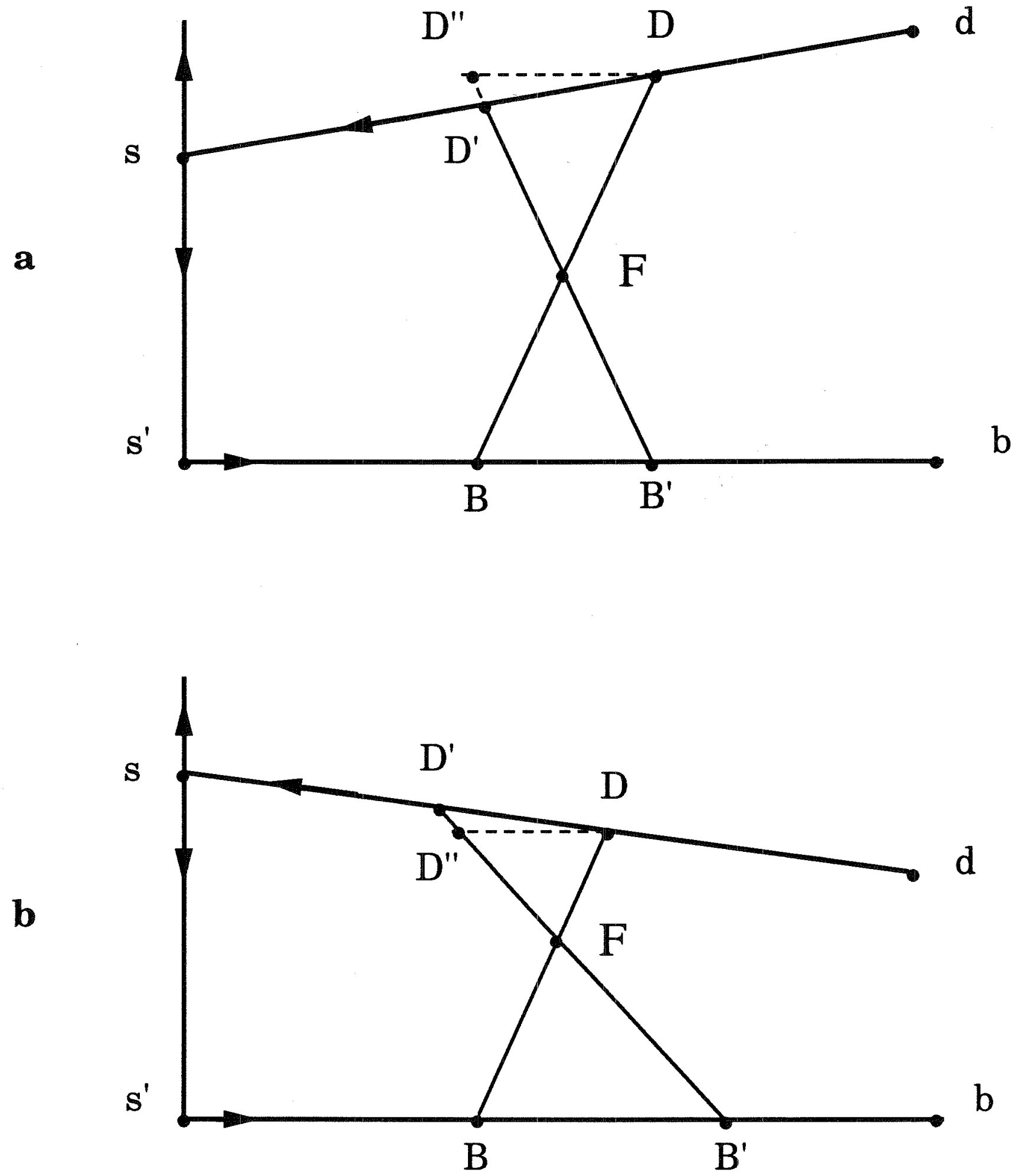

Figure 9: Geometry of the distillation region boundaries. a. $D$ increases b. $D$ decreases along the continuation path. 


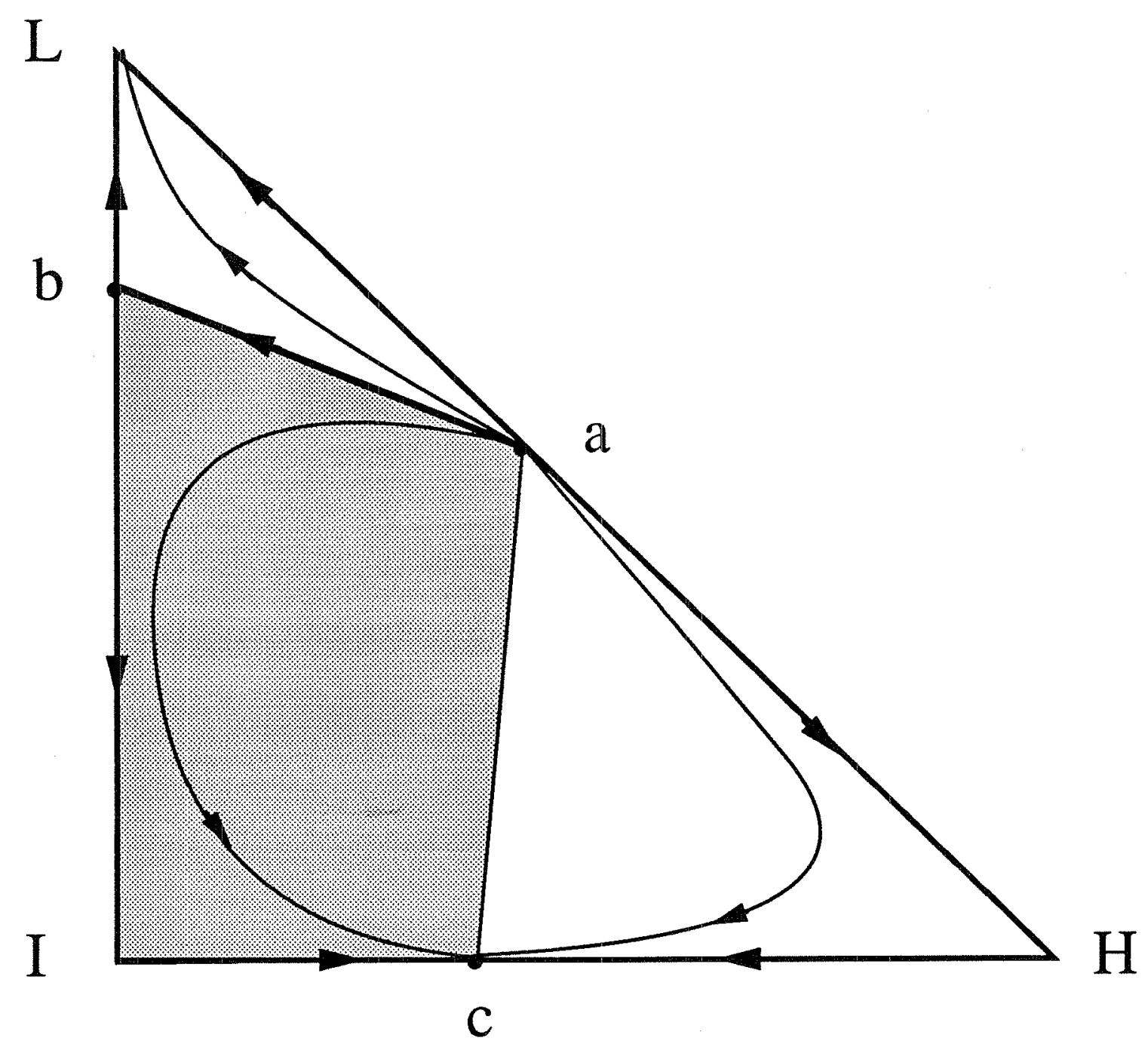

Figure 10: Residue curve diagram of a 231 class ternary mixture and the appropriate feed region. 


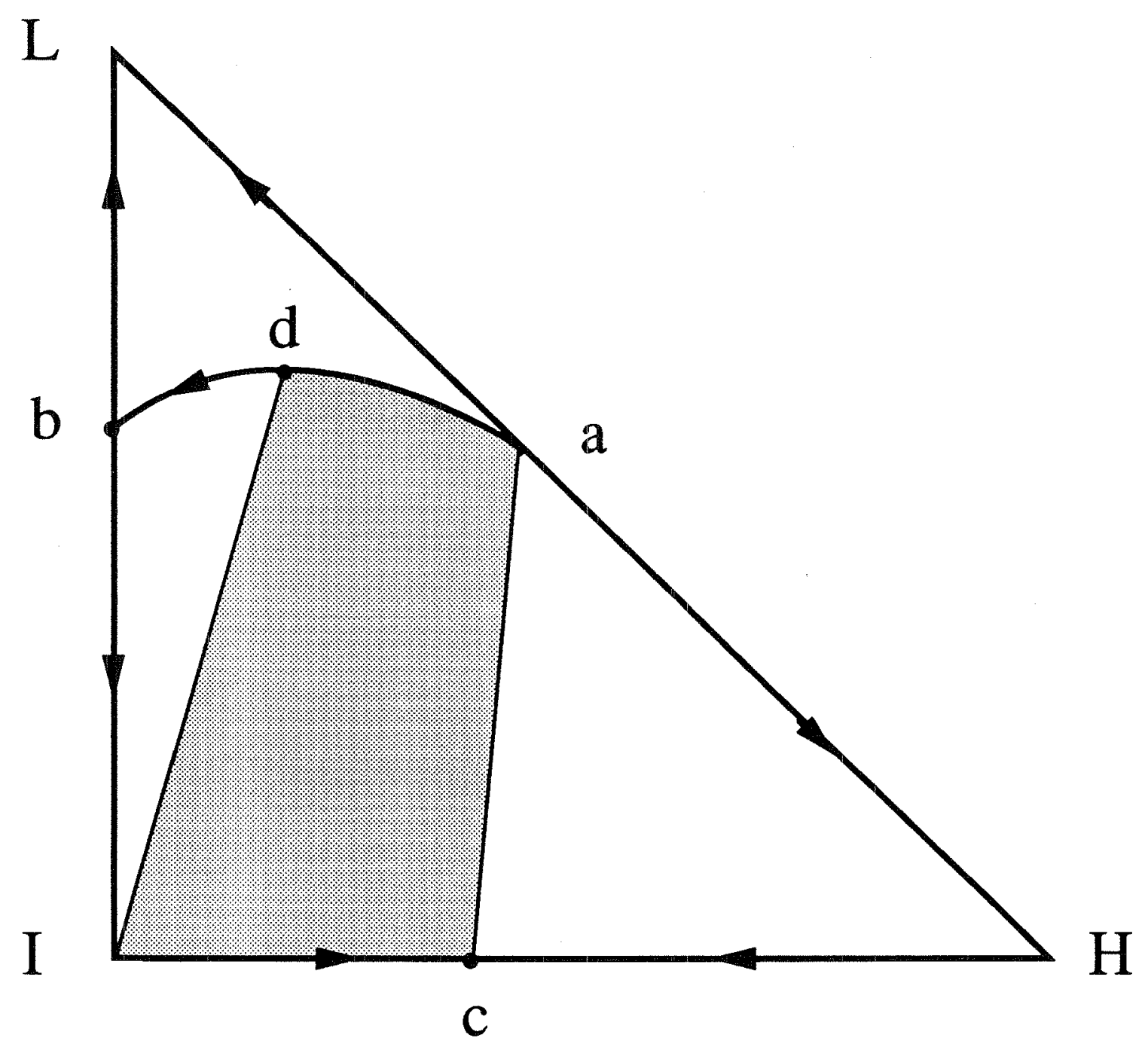

Figure 11: The curvature of the boundary affects the appropriate feed region. 


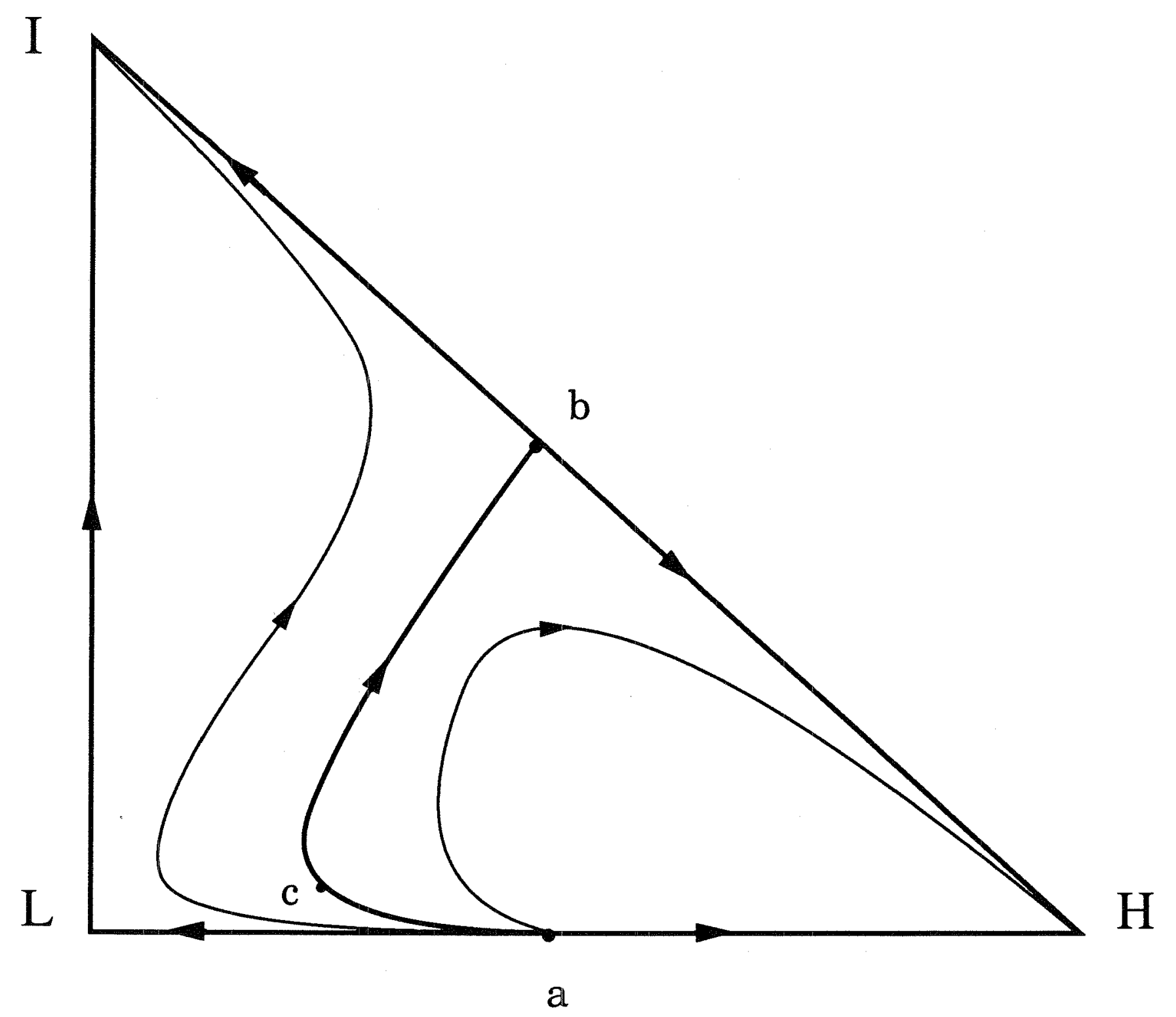

Figure 12a: Residue curve diagram of a 021 class ternary mixture that contains a highly curved boundary. 


$$
\begin{aligned}
& \mathrm{D}: \mathrm{b} \longrightarrow \mathrm{b} \rightarrow \mathrm{c}: \mathrm{i} \rightarrow \mathrm{F} \\
& \mathrm{B}: \mathrm{F} \rightarrow \gamma \rightarrow \delta \mapsto \mathrm{H} \longrightarrow \mathrm{H} \\
& \mathrm{F}_{\mathbf{D}}: 0 \uparrow \begin{array}{c:c}
\uparrow & \downarrow
\end{array} \mathrm{F}_{\mathbf{F}}
\end{aligned}
$$

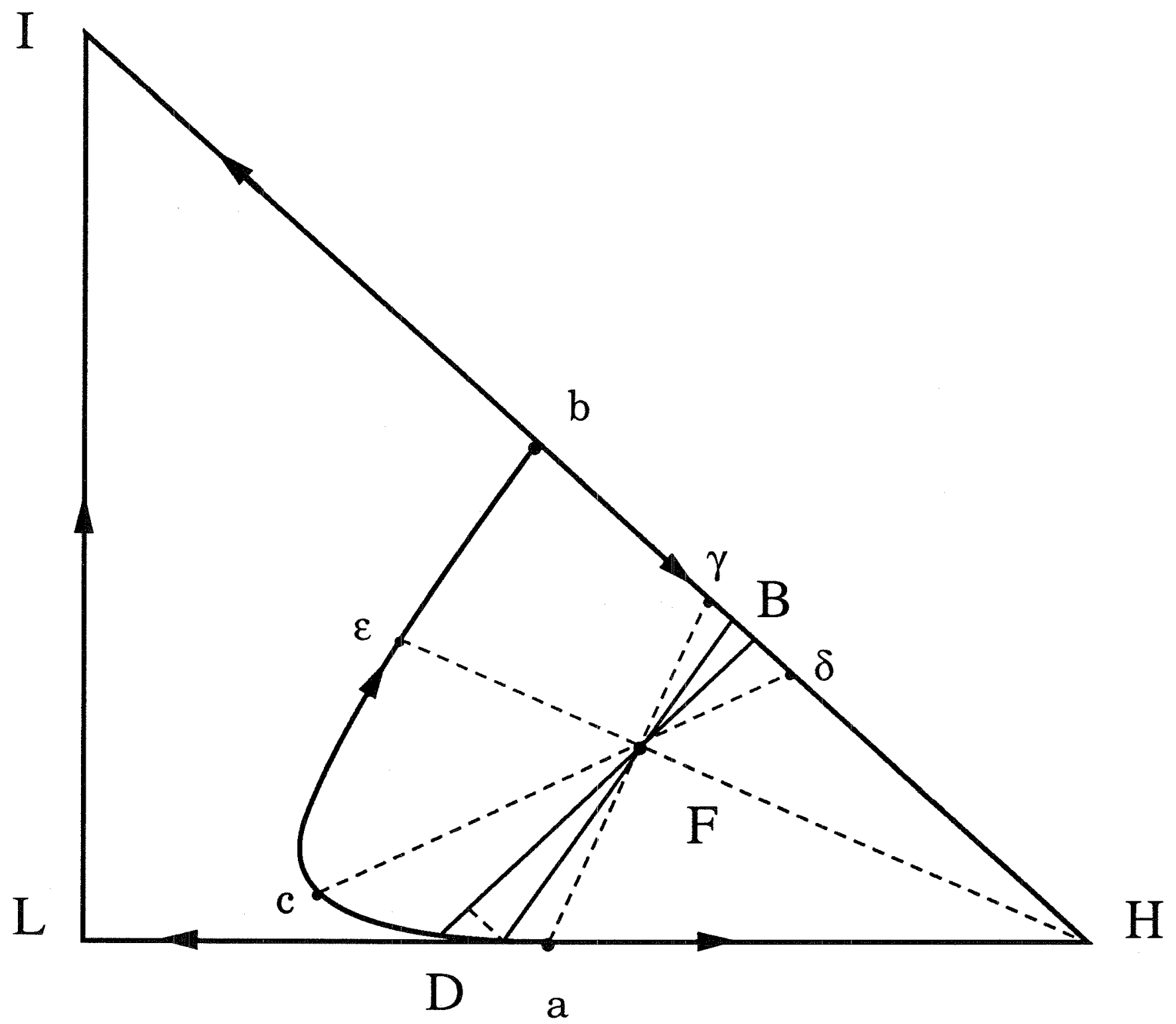

Figure 12b: Highly curved boundaries can induce multiplicities 


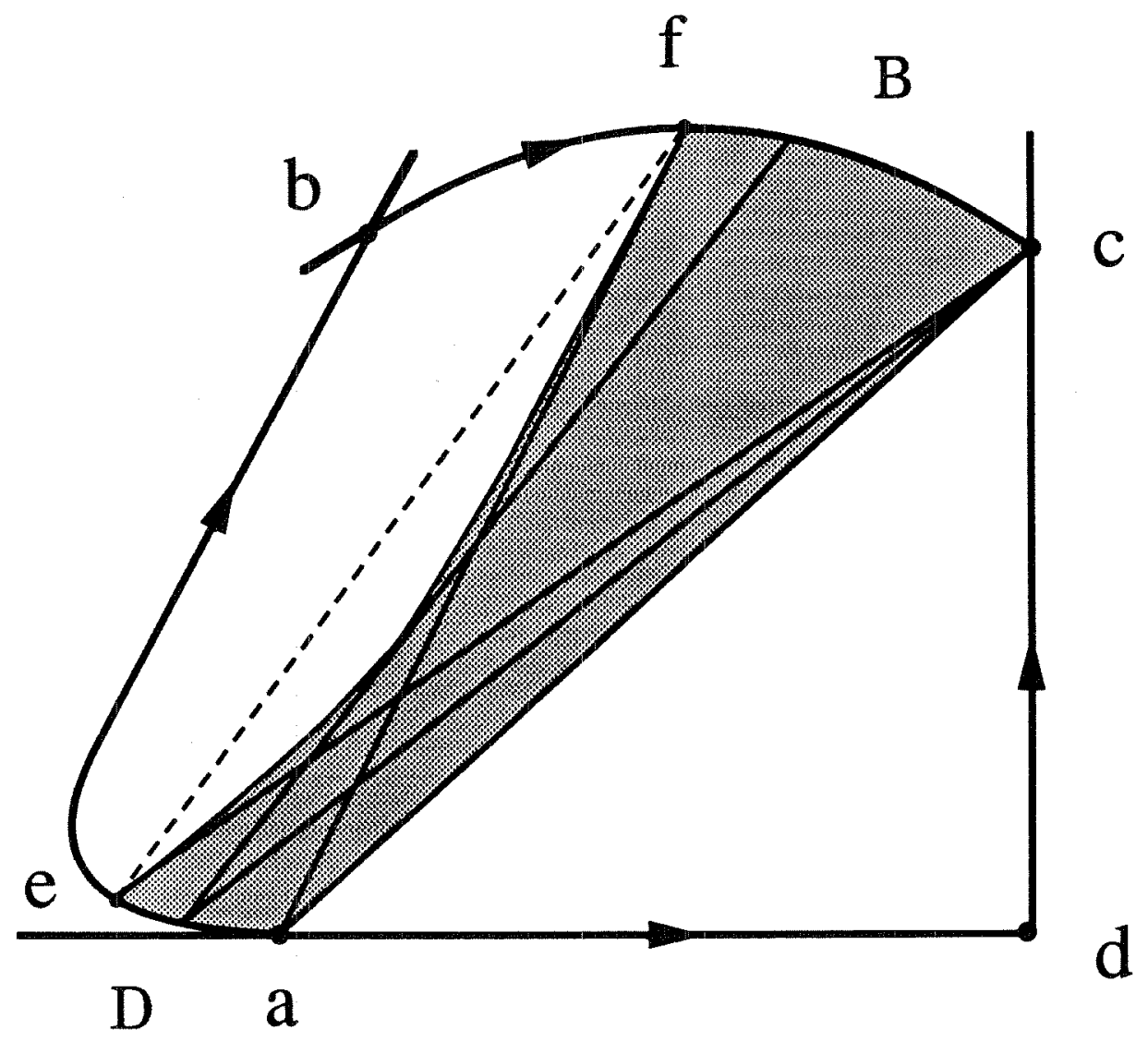

Figure 13: The appropriate feed region in the case of two curved boundaries. 
Concentration Entrainer feed Azeotropic feed

$\begin{array}{ccc}\text { Acetone (L) } & 0.0 & 0.90 \\ \text { Heptane (H) } & 0.0 & 0.10 \\ \text { Benzene (I-E) } & 1.0 & 0.0\end{array}$

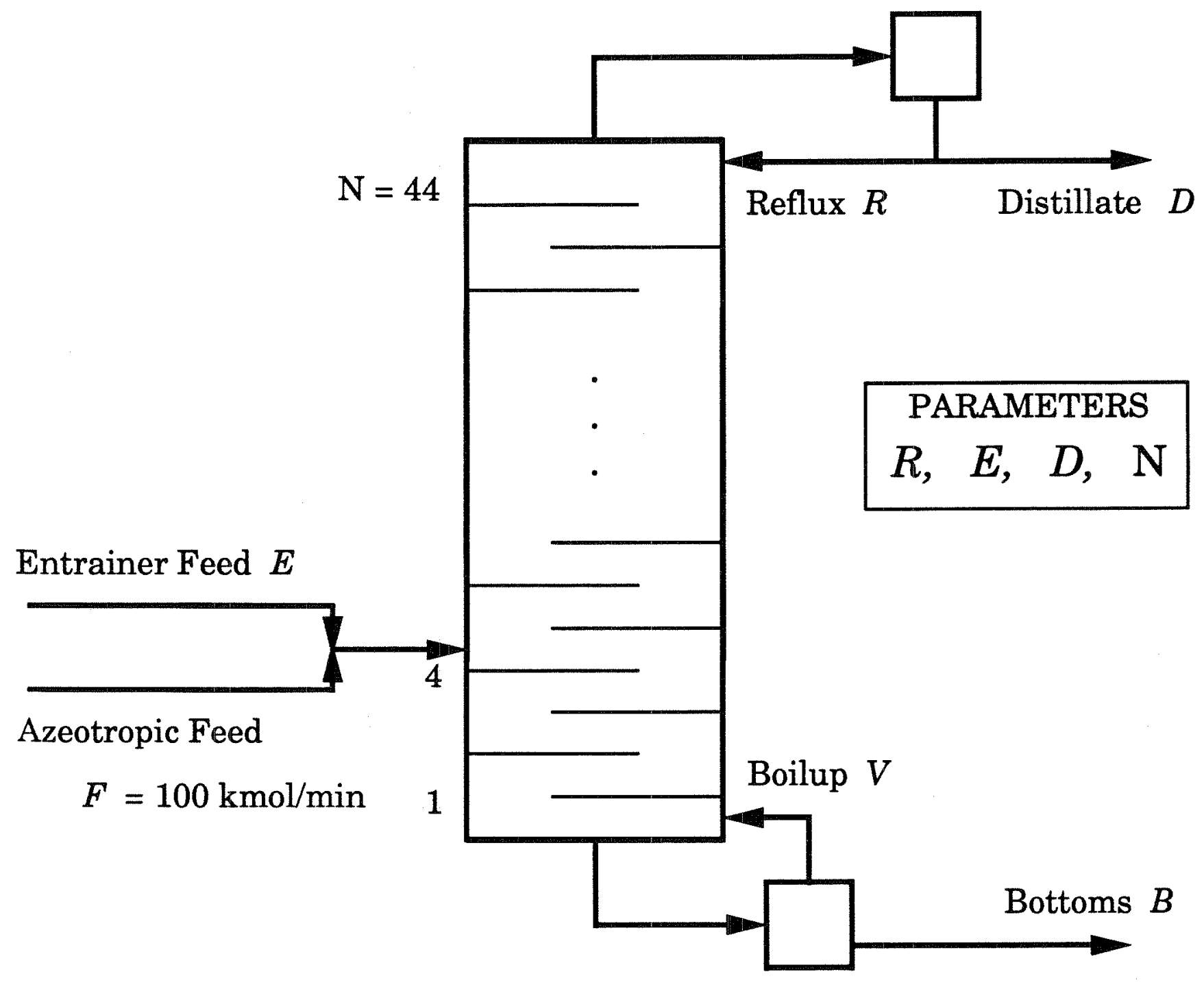

Figure 14: The acetone (L) - heptane $(\mathrm{H})$ - benzene (I-E) azeotropic column. 


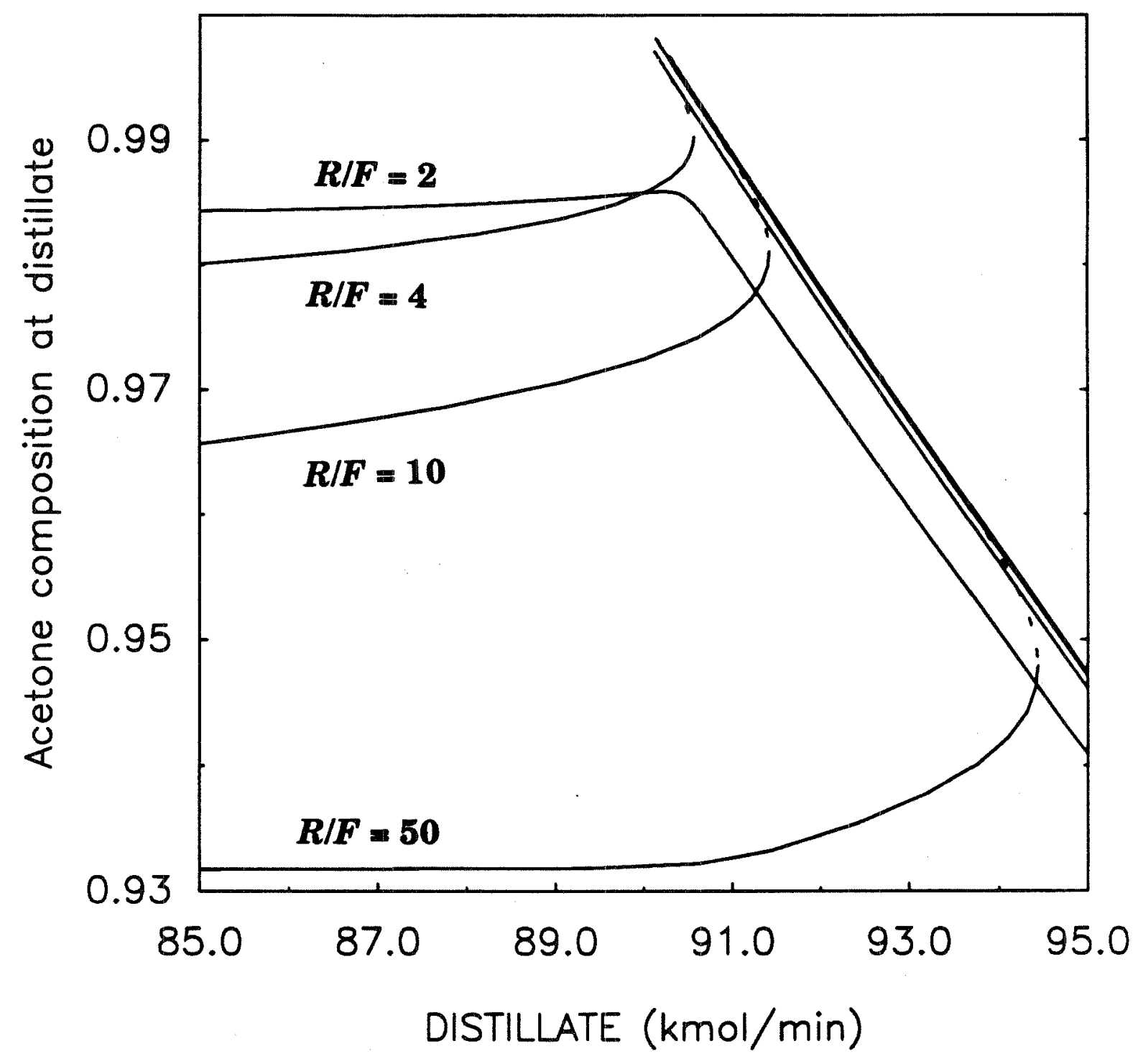

Figure 15: Bifurcation diagrams for a column with $\mathrm{N}=44$ trays, $E / F=1$ and various $R / F$. The distillate flow is the bifurcation parameter. 

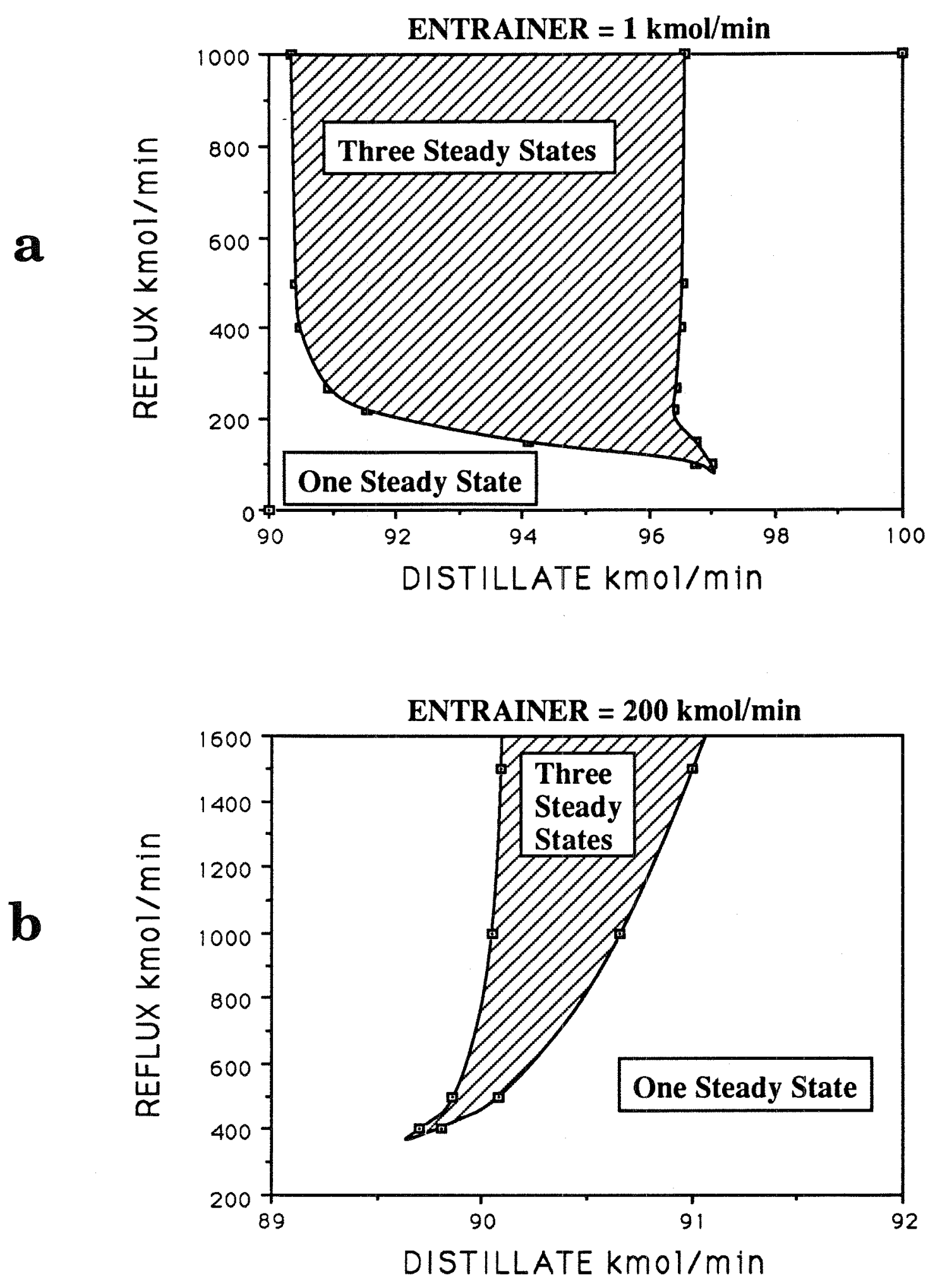

Figure 16: Reflux - Distillate Multiplicity Regions. 


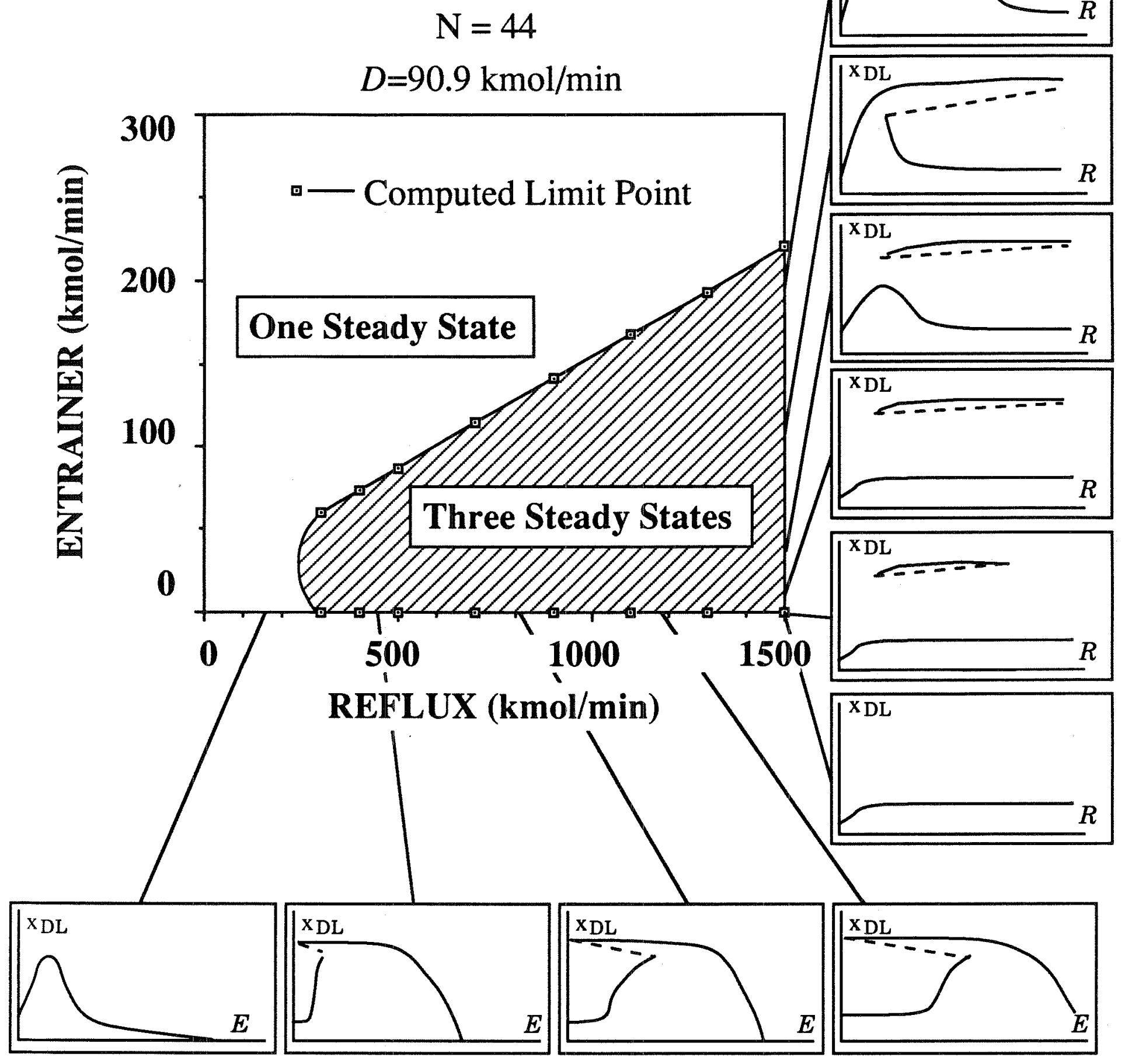

Figure 17: Entrainer - Reflux Multiplicity Region and typical bifurcation diagrams with the entrainer and reflux flows as the bifurcation parameters. 


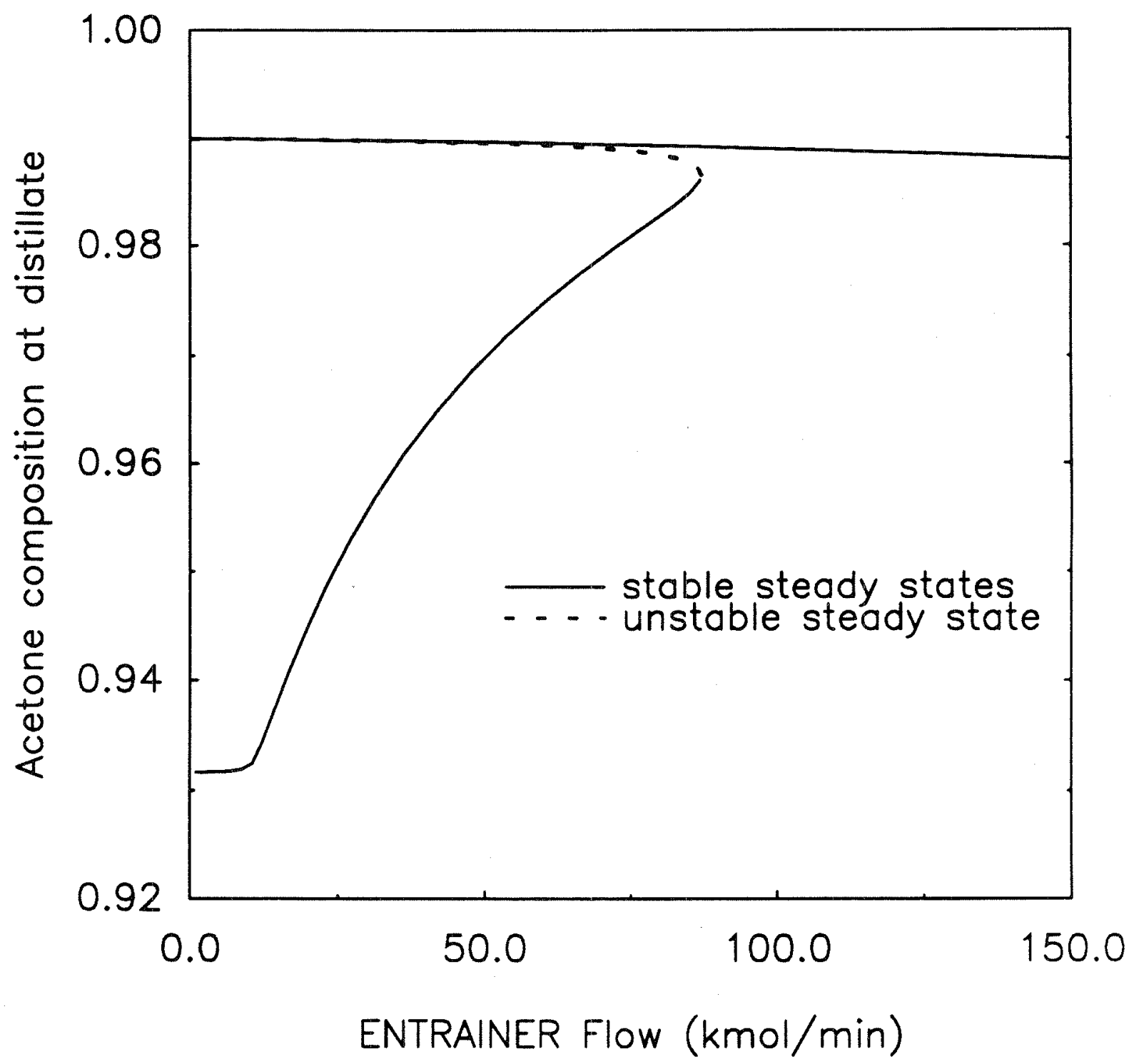

Figure 18: Bifurcation diagram for a column with $\mathrm{N}=44$ trays, $R=500 \mathrm{kmol} / \mathrm{min}$ and $D=90.9 \mathrm{kmol} / \mathrm{min}$. The entrainer feed flow is the bifurcation parameter. 


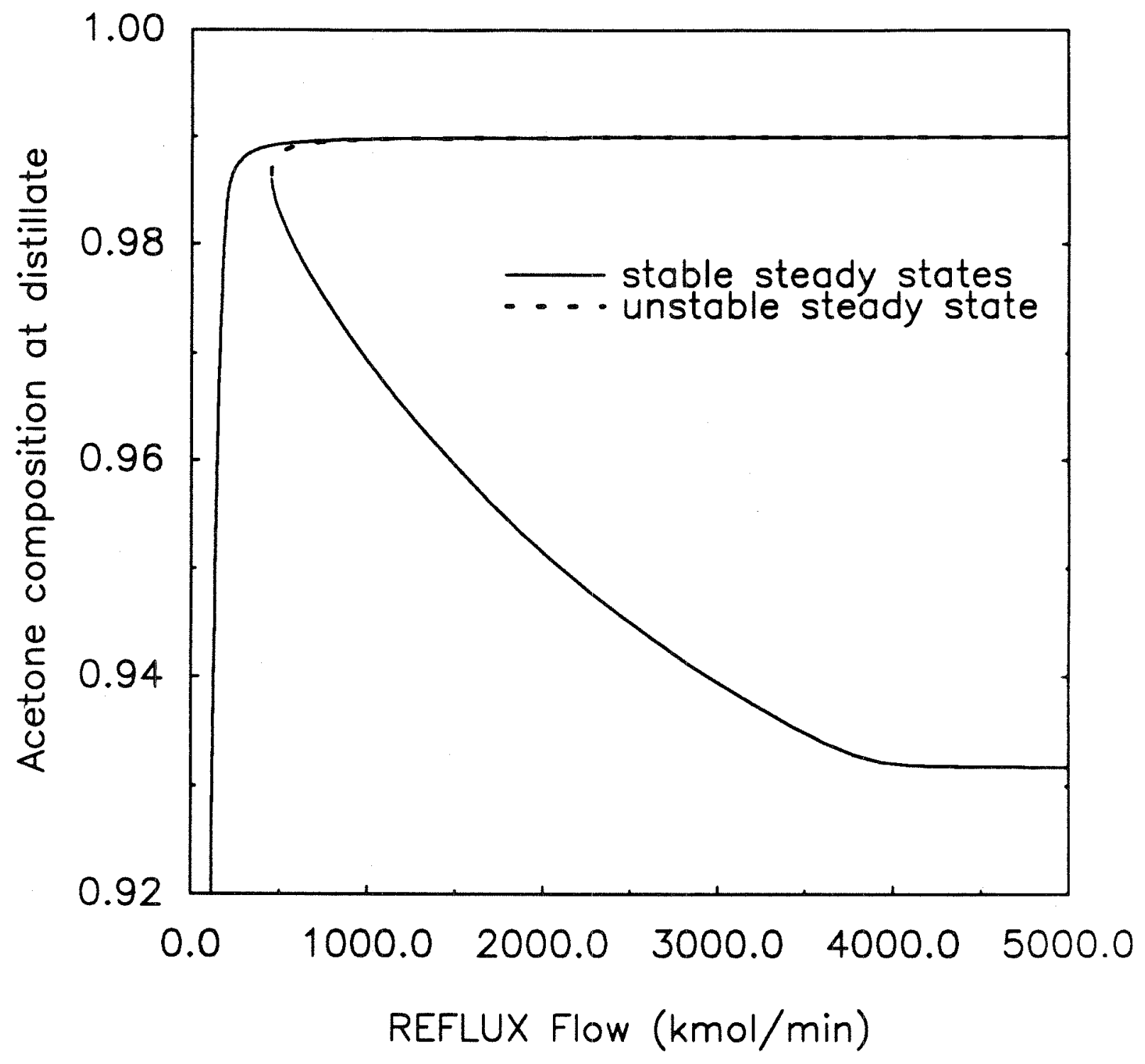

Figure 19: Bifurcation diagram for a column with $\mathrm{N}=44$ trays, $E=80 \mathrm{kmol} / \mathrm{min}$ and $D=90.9 \mathrm{kmol} / \mathrm{min}$. The reflux flow is the bifurcation parameter. 


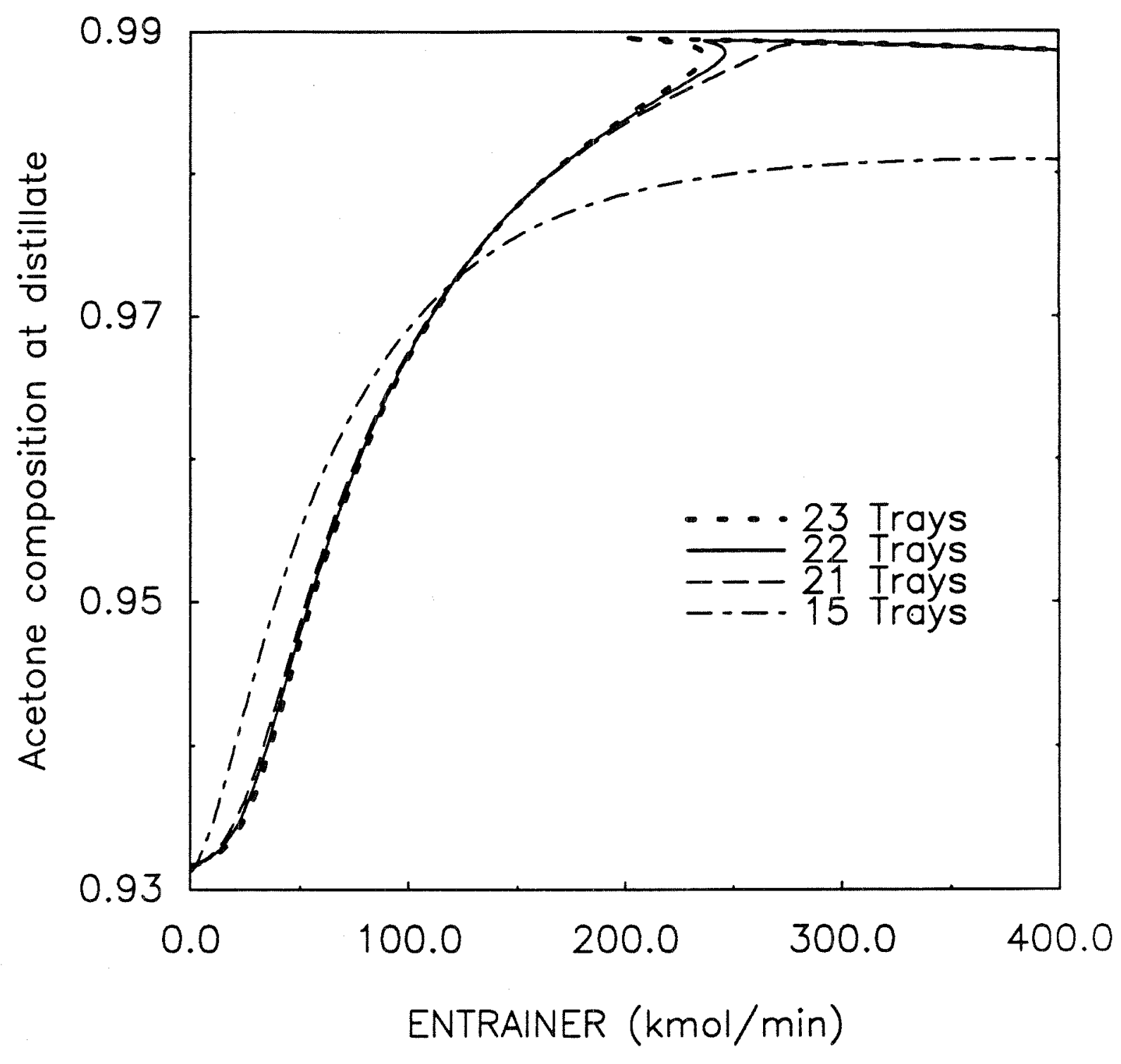

Figure 20: Vanishing multiplicity in small columns with $R=1500 \mathrm{kmol} / \mathrm{min}$ and $D=90.9 \mathrm{kmol} / \mathrm{min}$. 


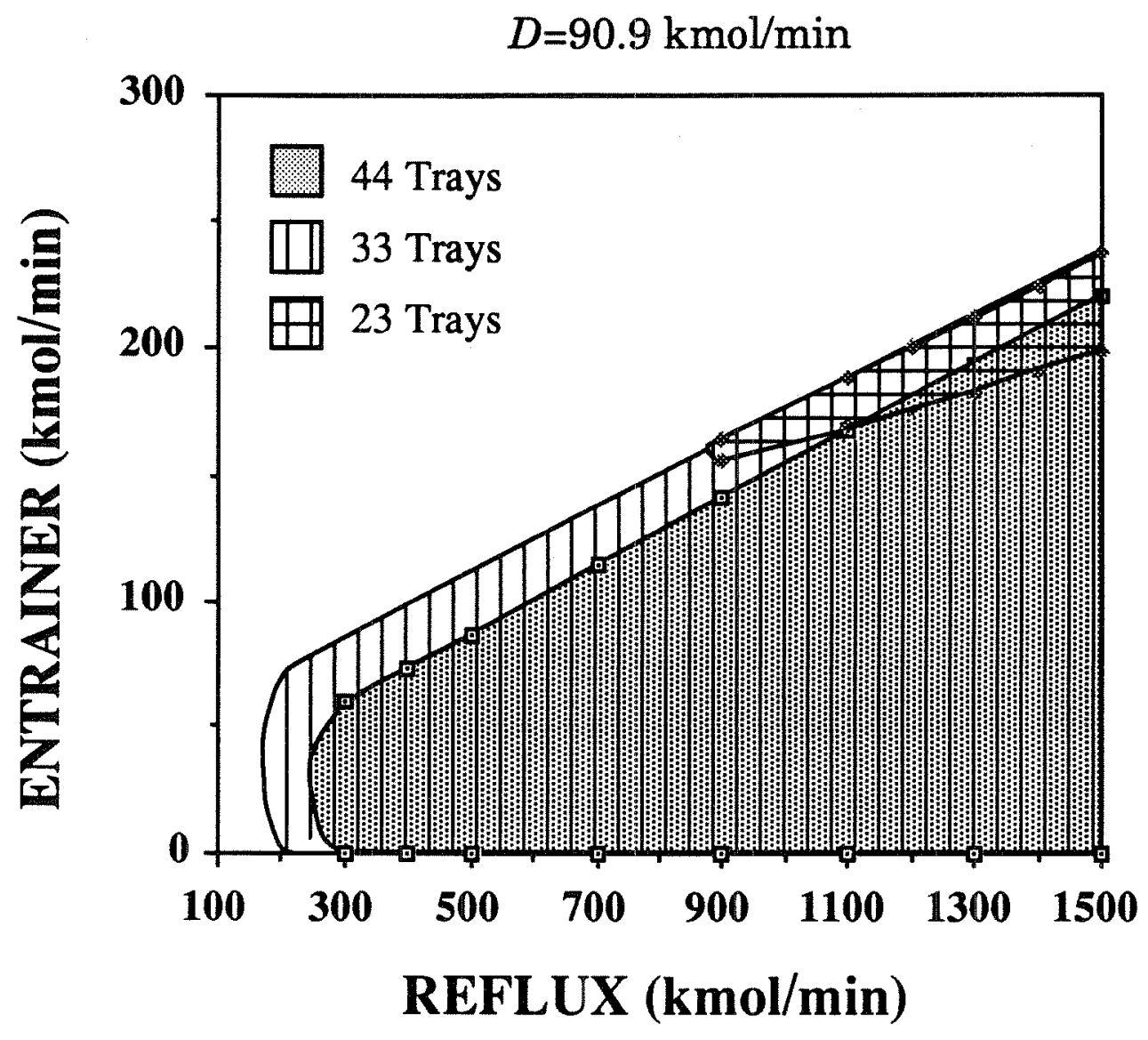

Figure 21: Entrainer - Reflux multiplicity region variation with the Number of Trays. 


\section{Acetone}

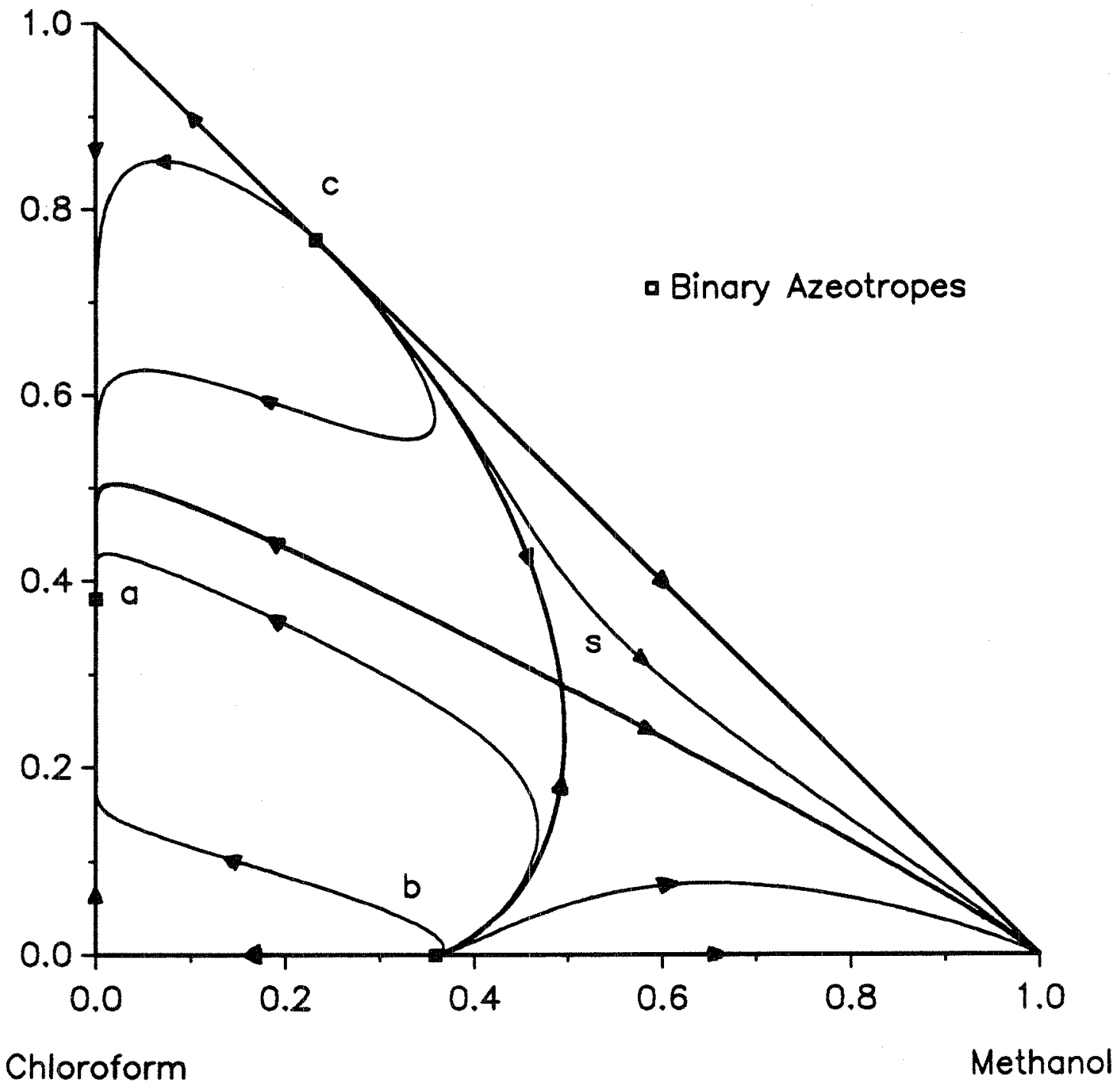

Figure 22a: The acetone - methanol - chloroform residue curve diagram. 


\section{Acetone}

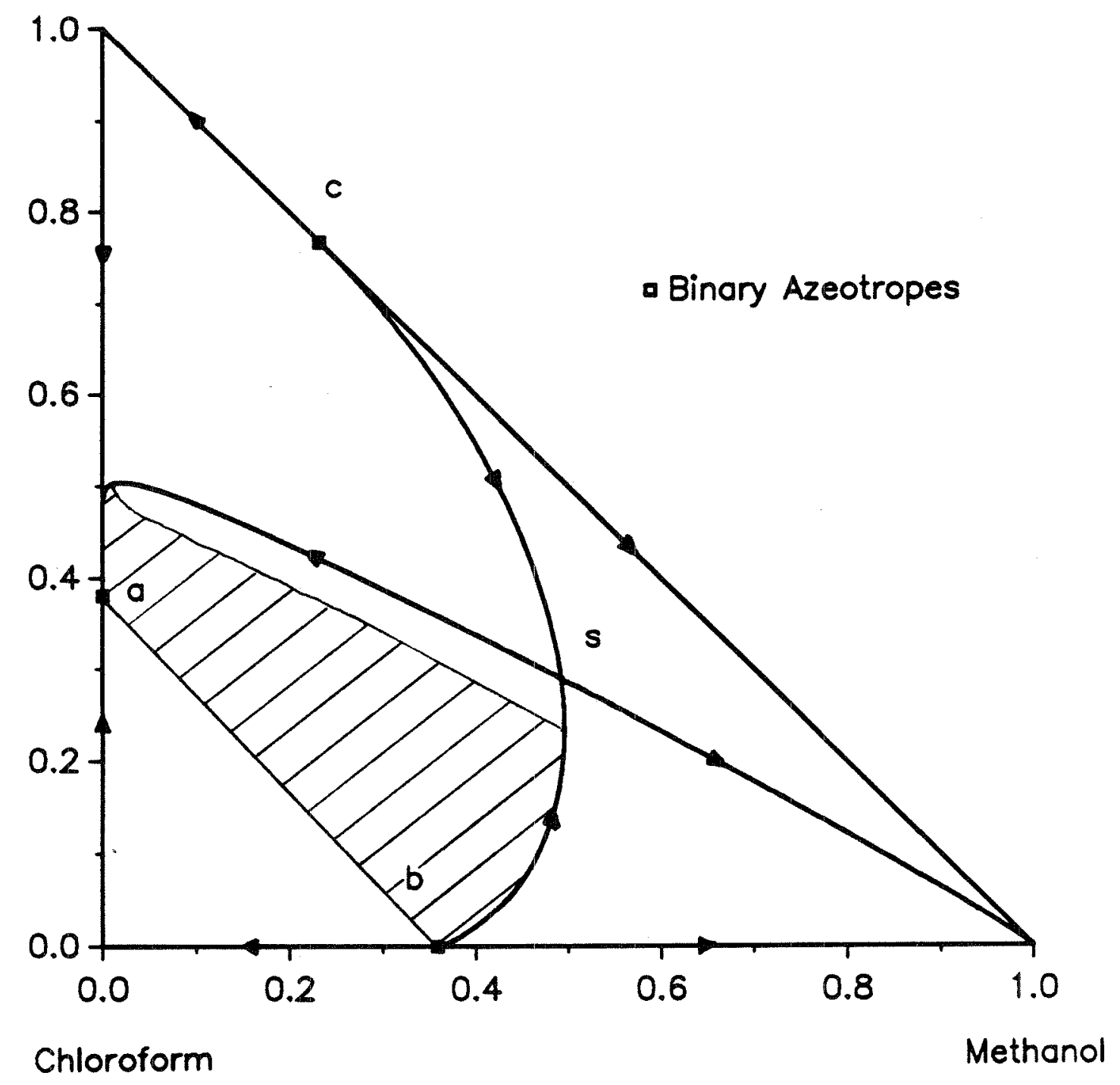

Figure 22b: The four distillation regions and the appropriate feed region in the acetone - methanol chloroform composition triangle. 


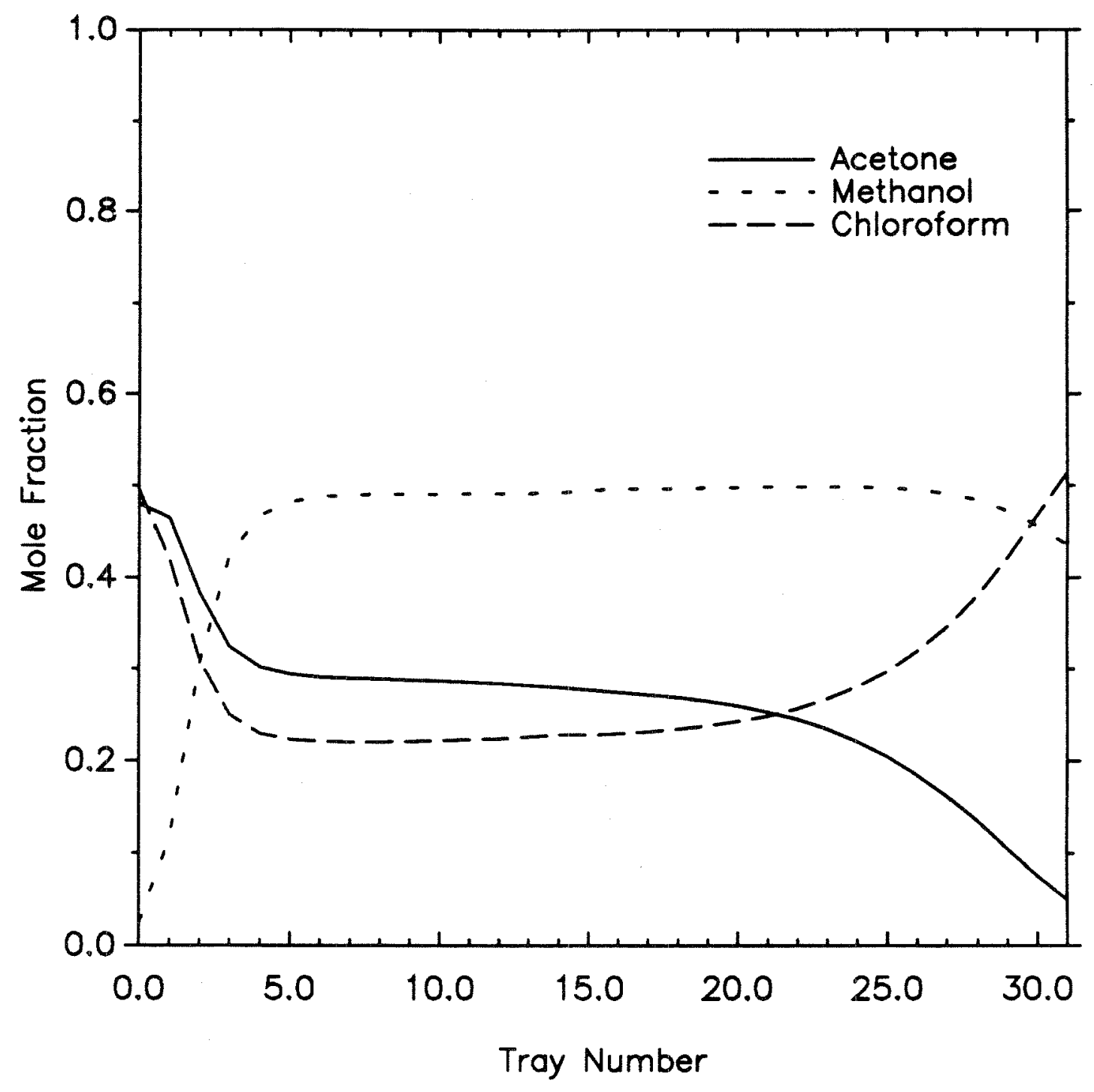

Figure 23a: First stable profile of a column with $\mathrm{N}=30$ trays, $E / F=.5$ and $R / F=100$. 


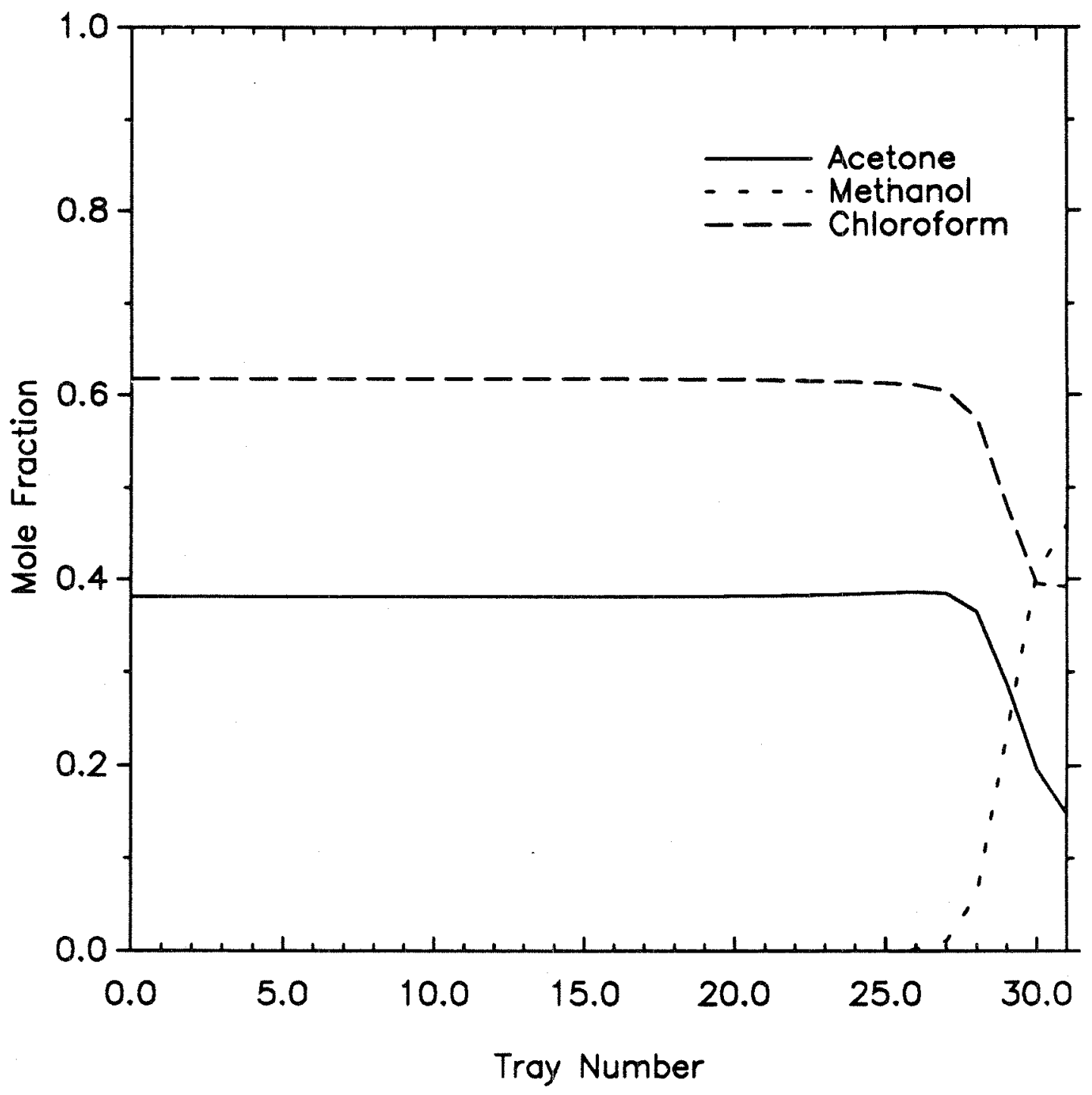

Figure 23b: Second stable profile of a column with $\mathrm{N}=30$ trays, $E / F=.5$ and $R / F=100$. 


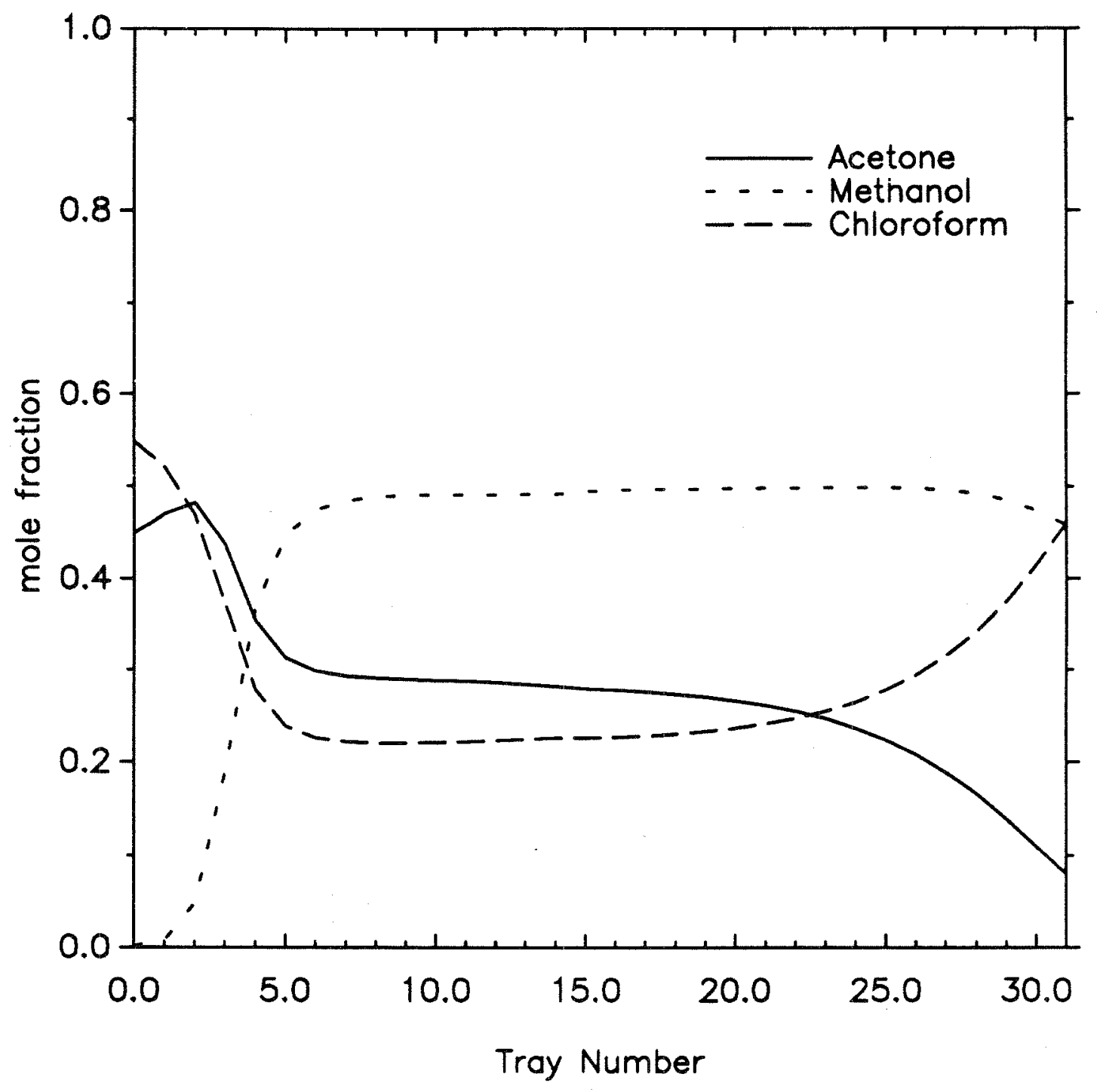

Figure 23c: Unstable profile of a column with $\mathrm{N}=30$ trays, $E / F=.5$ and $R / F=100$. 
Acetone

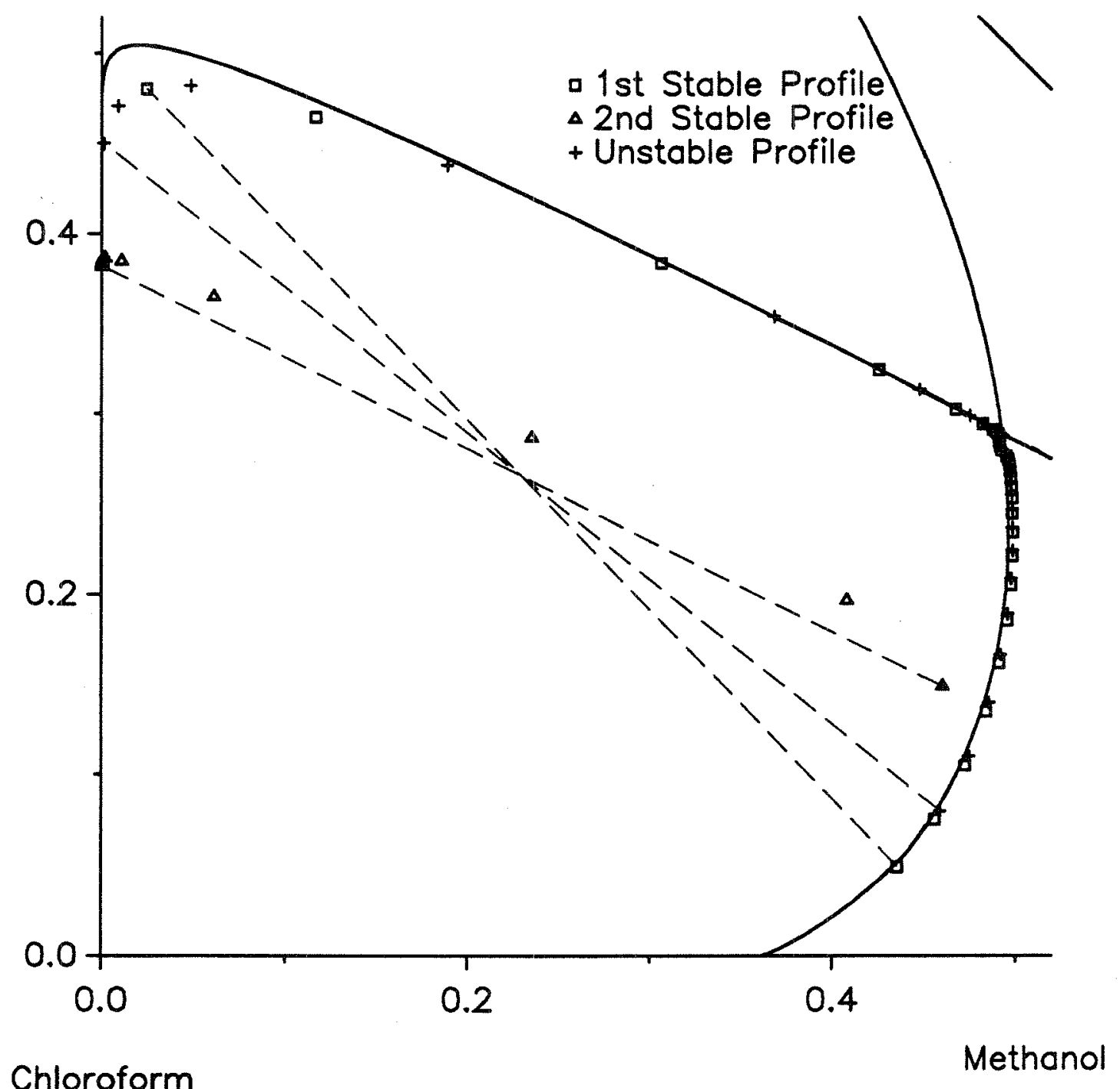

Chloroform

Figure 23d: The three steady states of the Acetone / Methanol / Chloroform column with $\mathrm{N}=30$ trays, $E / F=.5$ and $R / F=100$. in the composition triangle. 


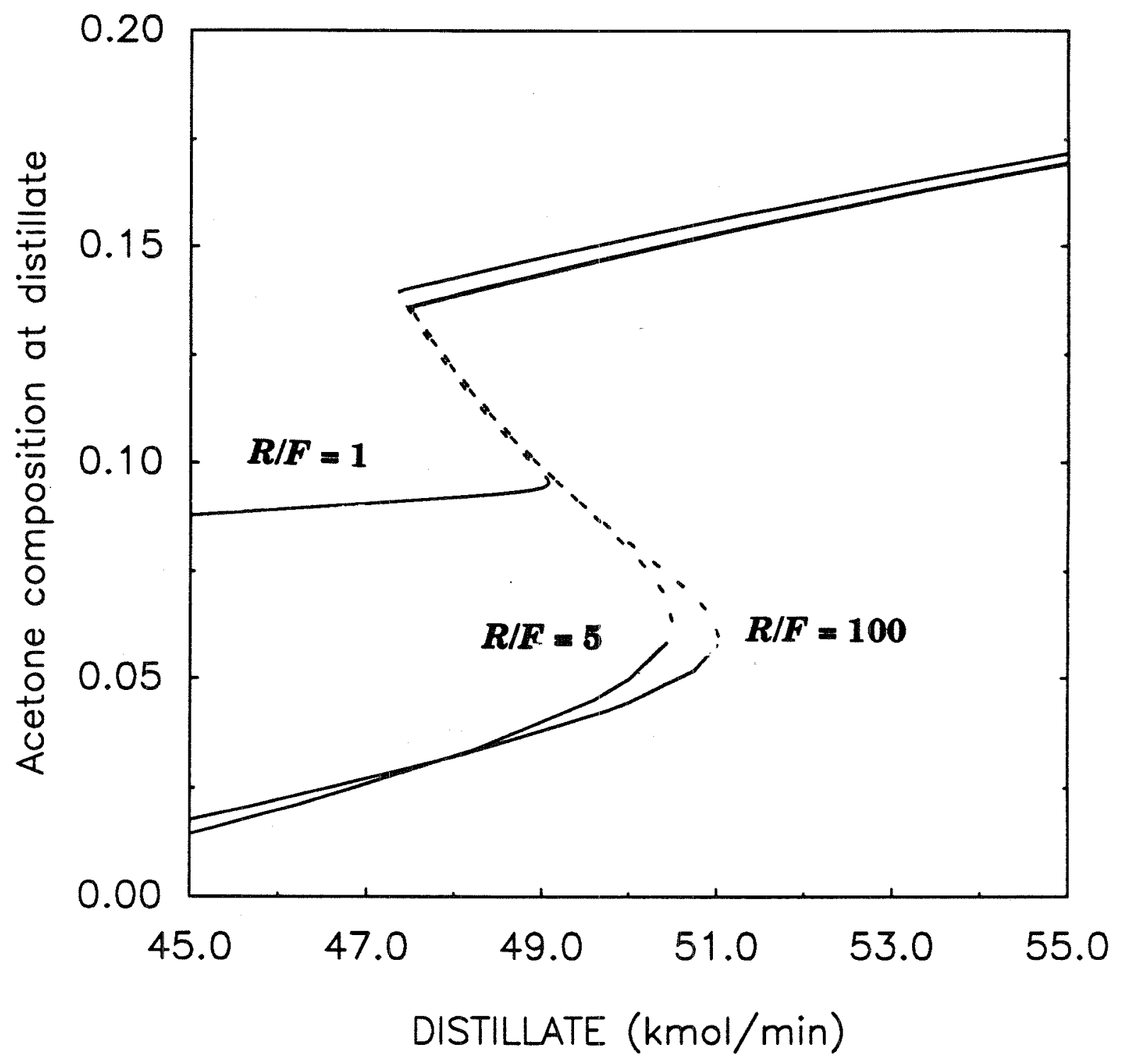

Figure 24: Bifurcation diagrams for the Acetone / Methanol / Chloroform column with $\mathrm{N}=30$ trays and various $R / F$. The distillate flow is the bifurcation parameter. 


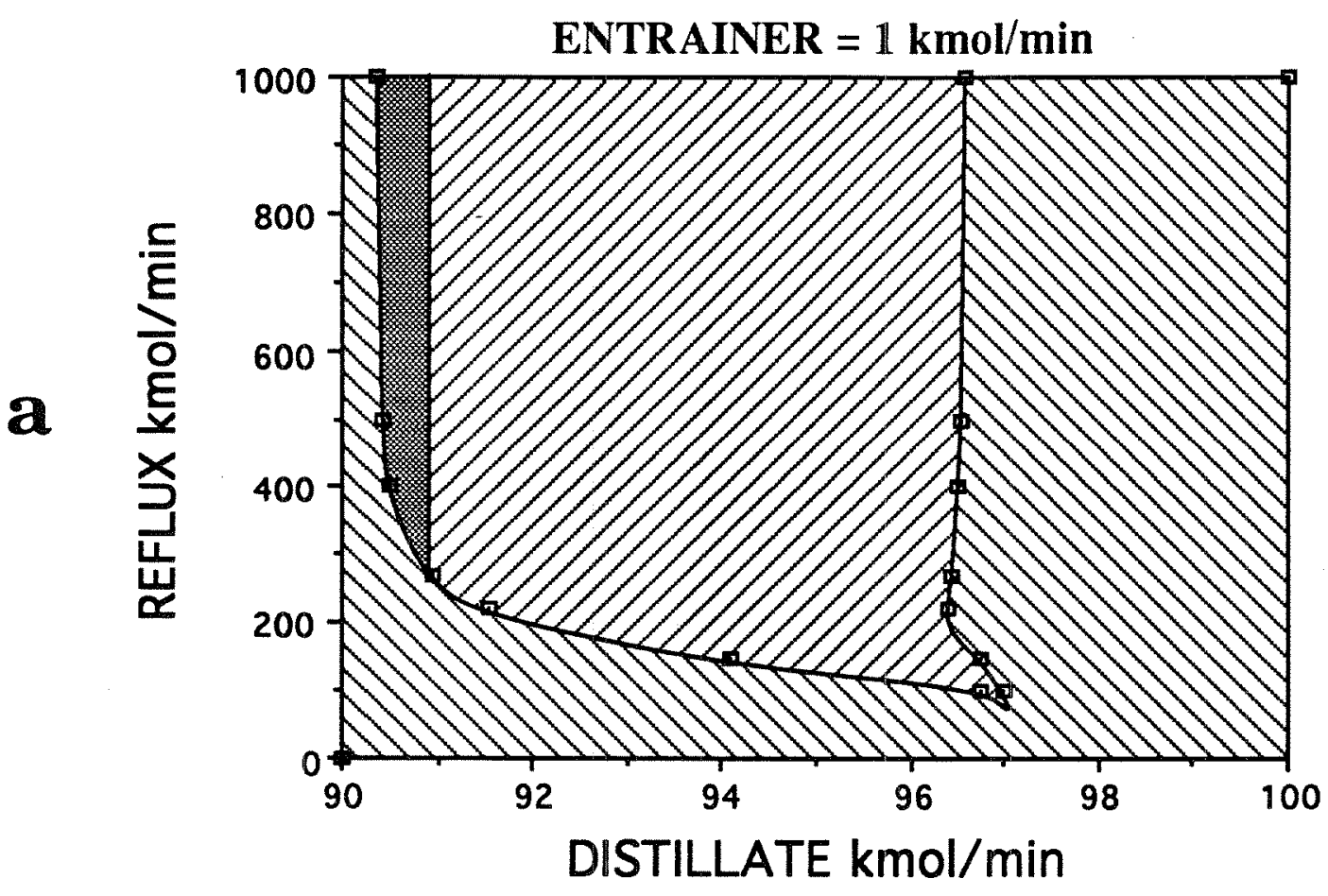

\begin{tabular}{|c|}
\hline SPECS \\
99\% PURITY \\
99\% RECOVERY \\
\hline
\end{tabular}

ENTRAINER $=200 \mathrm{kmol} / \mathrm{min}$

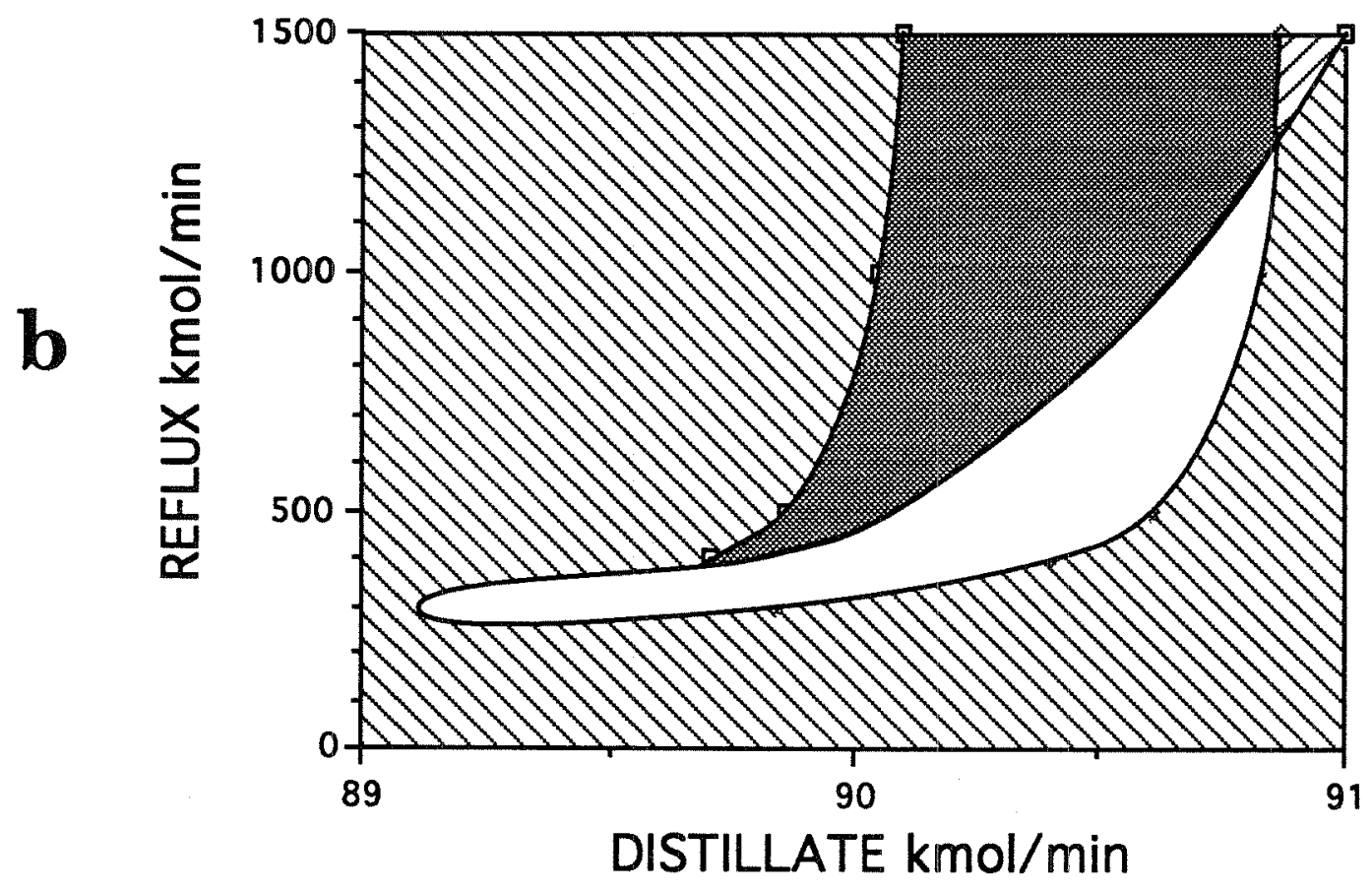

$\square$ S.S. \& Specs

3 S.S. \& Specs

$\square$ 3 s.S.

$\triangle 1$ S.S.

Figure 25: Reflux - Distillate Multiplicity and Specifations Regions. 


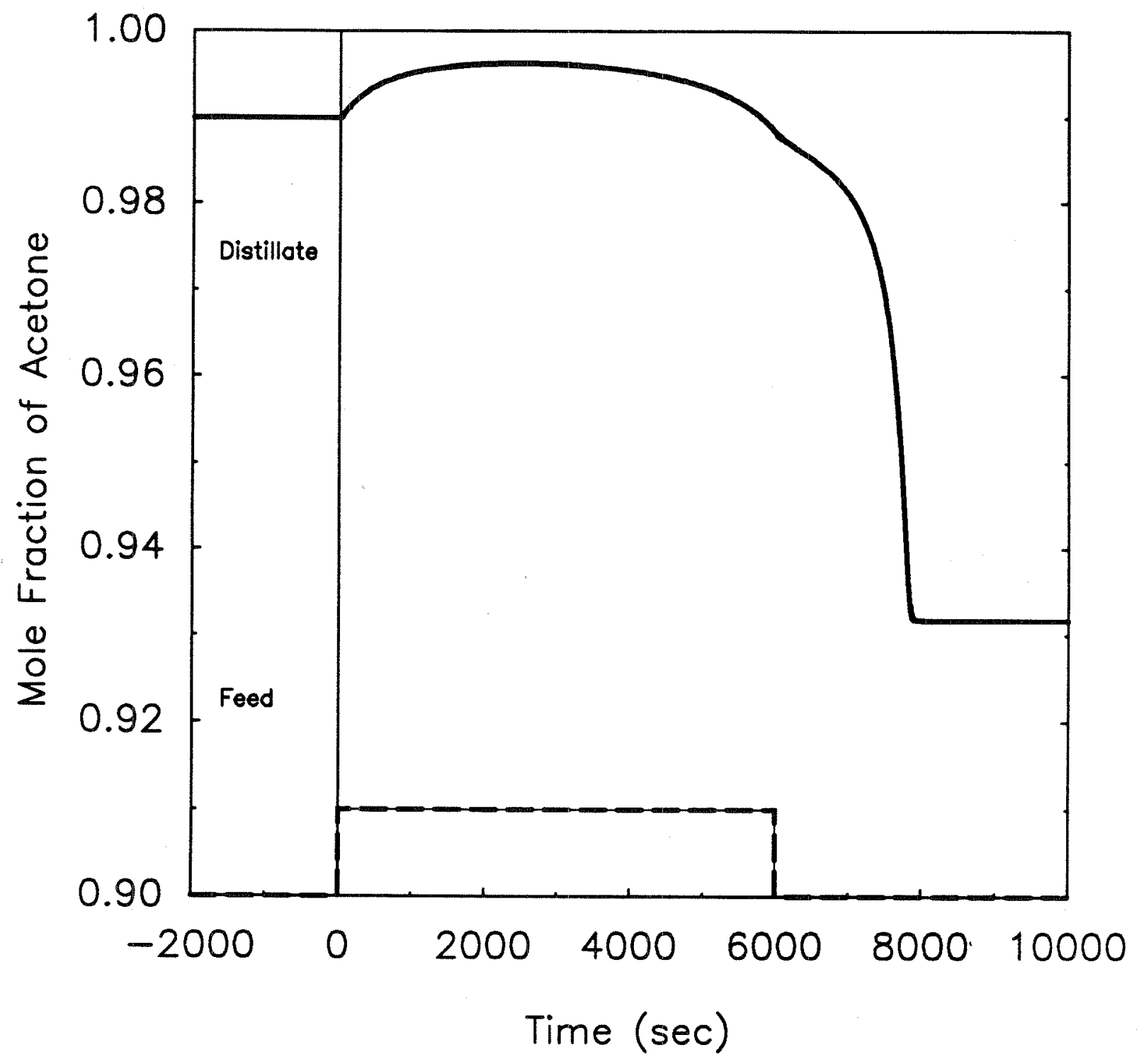

Figure 26: Open - loop dynamical behavior under a feed composition disturbance. 
Table A1: Antoine coefficients for the components used in the simulations.

\begin{tabular}{|l|l|l|l|}
\cline { 2 - 4 } \multicolumn{1}{c|}{} & \multicolumn{1}{c|}{$\mathrm{A}$} & $\mathrm{B}$ & $\mathrm{C}$ \\
\hline Heptane & 20.7664 & -2911.32 & -56.514 \\
\hline Benzene & 20.7936 & -2788.51 & -52.360 \\
\hline Acetone & 21.3099 & -2801.53 & -42.875 \\
\hline Methanol & 23.4832 & -3634.01 & -33.768 \\
\hline Chloroform & 20.865 & -2696.9 & -46.16 \\
\hline
\end{tabular}

Table A2: Van Laar coefficients for the acetone - heptane - benzene mixture.

\begin{tabular}{|c|c|c|c|}
\hline $\mathrm{i}$ & Heptane & Benzene & Acetone \\
\hline Heptane & 0 & 159.20 & 569.40 \\
\hline Benzene & 112.00 & 0 & 144.20 \\
\hline Acetone & 444.80 & 189.60 & 0 \\
\hline
\end{tabular}

Table A3: Van Laar coefficients for the acetone - methanol - chloroform mixture.

\begin{tabular}{|l|c|c|c|}
\hline $\mathrm{i}$ & Acetone & Methanol & Chloroform \\
\hline Acetone & 0 & 205.40 & -260.23 \\
\hline Methanol & 232.00 & 0 & 597.03 \\
\hline Chloroform & -234.17 & 313.06 & 0 \\
\hline
\end{tabular}

